\title{
INFINITELY GENERATED FREE NILPOTENT GROUPS: COMPLETENESS OF THE AUTOMORPHISM GROUPS
}

\author{
VLADIMIR TOLSTYKH
}

\begin{abstract}
We transfer the results of Dyer, Formanek and Kassabov on the automorphism towers of finitely generated free nilpotent groups to infinitely generated free nilpotent groups. We prove that the automorphism groups of infinitely generated free nilpotent groups are complete. By combining the results of Dyer, Formanek, Kassabov with the results in the present paper, one gets that the automorphism tower of any free nilpotent group terminates after finitely many steps.
\end{abstract}

\section{INTRODUCTION}

Baumslag conjectured in the 1970s that the automorphism tower of a finitely generated free group (free nilpotent group) must be very short. Dyer and Formanek [8] justified the conjecture concerning finitely generated free groups in the "sharpest sense" by proving that the automorphism group $\operatorname{Aut}\left(F_{n}\right)$ of a non-abelian free group $F_{n}$ of finite rank $n$ is complete. Recall that a group $G$ is said to be complete if $G$ is centreless and all automorphisms of $G$ are inner; it then follows that $\operatorname{Aut}(G) \cong G$. Thus $\operatorname{Aut}\left(\operatorname{Aut}\left(F_{n}\right)\right) \cong \operatorname{Aut}\left(F_{n}\right)$, or, in other words, the height of the automorphism tower over $F_{n}$ is two. The proof of completeness of $\operatorname{Aut}\left(F_{n}\right)$ given by Dyer and Formanek in [8] has later been followed by the proofs given by Formanek [1], by Khramtsov [14, by Bridson and Vogtmann [3, and by the author [17. The proof given in [17] works for arbitrary non-abelian free groups; thus the automorphism groups of infinitely generated free groups are also complete.

Let $F_{n, c}$ denote a free nilpotent group of finite rank $n \geqslant 2$ and of nilpotency class $c \geqslant 2$. In 9 Dyer and Formanek studied the automorphism towers of free nilpotent groups $F_{n, 2}$ of class two. They showed that the group $\operatorname{Aut}\left(F_{n, 2}\right)$ is complete provided that $n \neq 3$. In the case when $n=3$ the height of the automorphism tower of $F_{n, 2}$ is three. The main result of [18] states that the automorphism group of any infinitely generated free nilpotent group of class two is complete.

In [10] Dyer and Formanek proved completeness of the automorphism groups of groups of the form $F_{n} / R^{\prime}$ where $R$ is a characteristic subgroup of $F_{n}$ which is contained in the commutator subgroup $F_{n}^{\prime}$ of $F_{n}$ and $F_{n} / R$ is residually torsion-free nilpotent.

In his Ph. D. thesis [13] Kassabov found an upper bound $u(n, c) \in \mathbf{N}$ for the height of the automorphism tower of $F_{n, c}$ in terms of $n$ and $c$, thereby finally proving Baumslag's conjecture on finitely generated free nilpotent groups. By analyzing the function $u(n, c)$ one can conclude that if $c$ is small compared to $n$, then the height of the automorphism tower of $F_{n, c}$ is at most three.

2000 Mathematics Subject Classification. 20F28 (20F18, 03C60). 
The main result of the present paper generalizing the main result of [18] states that the automorphism group of any infinitely generated free nilpotent group of nilpotency class $\geqslant 2$ is complete.

We would like, before discussing the structure of the paper, to discuss modeltheoretic terminology that will be used throughout the paper. Generally speaking, it is often required, when studying the automorphisms of a given structure $\mathcal{M}$, to show that a certain subset (relation) $S$ of $\mathcal{M}$ is invariant under all automorphisms of $\mathcal{M}$. Similarly, it might be required to show that $S$ is fixed setwise by all automorphisms of $\mathcal{M}$ that fix a given subset $R$ of $\mathcal{M}$ pointwise. Natural examples of subsets of $\mathcal{M}$ that are invariant under all automorphisms are given by subsets that are definable in $\mathcal{M}$ by formulae of some logic $\mathcal{L}$. Let $\mathcal{L}$ be a logic and $\mathcal{M}$ a structure in the language of $\mathcal{L}$. A subset $S$ of $\mathcal{M}$ is said to be definable in $\mathcal{M}$ by means of $\mathcal{L}$, if there is a formula $\chi(v)$ of $\mathcal{L}$ such that $S=\chi(\mathcal{M})$, that is, $S$ is the set of all realizations ("solutions") of $\chi$ in $\mathcal{M} ; S$ is said to be definable with parameters $\bar{a}$ where $\bar{a}$ is a tuple of elements of $\mathcal{M}$ if $S=\vartheta(\mathcal{M}, \bar{a})$ for some formula $\vartheta(v, \bar{u})$ of $\mathcal{L}$.

Proposition 0.1. [12, Lemma 2.1.1] Suppose that a subset $S$ is definable in $\mathcal{M}$ (resp. definable in $\mathcal{M}$ with parameters $\bar{a}$ ) by means of some logic $\mathcal{L}$. Then $S$ is fixed setwise by all automorphisms of $\mathcal{M}$ (resp. by all automorphisms of $\mathcal{M}$ that fix parameters $\bar{a}$ elementwise).

For instance, the family $i(G)$ of all involutions is definable in any group $G$ by means of both first-order and second-order logics (for the definition of the full second-order logic see Section 2.8 of [12]); on the other hand, the subgroup generated by $i(G)$ may not be first-order definable in $G$, but it is second-order definable in $G$ etc. In most of cases below, we prefer to speak simply of definable subsets, skipping references to logics whose formulae could be used to define them, although it is safe to think that everywhere below "definable" means "second-order definable" (we must warn the reader that in model-theoretic texts "definable" often means "first-order definable").

We would like to stress that despite the presence of model-theoretic terminology in the paper, no prior knowledge of model theory is actually assumed, since the reader who is not familiar with model theory can always substitute his or her own arguments to see that a particular definable set behaves as in the conclusion of the Proposition above.

The paper is organized as follows. Let $N$ be an infinitely generated free nilpotent group of nilpotency class at least two and let $\Delta \in \operatorname{Aut}(\operatorname{Aut}(N))$. In the first section we show that the subgroup $\operatorname{Inn}(N)$ of all inner automorphisms of $N$ is definable in the group $\operatorname{Aut}(N)$. This implies that $\Delta$ can be followed by an inner automorphism $T_{\rho}$ of the group $\operatorname{Aut}(N)$ so that the resulting automorphism $\Delta_{1}=T_{\rho} \circ \Delta$ fixes the subgroup $\operatorname{Inn}(N)$ pointwise. The main result of the second section states that the subgroup $\mathrm{IA}_{2}(N)$, the kernel of the homomorphism $\operatorname{Aut}(N) \rightarrow \operatorname{Aut}(N /[N, N, N])$, determined by the natural homomorphism $N \rightarrow N /[N, N, N]$, is also fixed pointwise by $\Delta_{1}$; this constitutes the major step towards the proof that $\Delta_{1}$ fixes all elements of the subgroup IA $(N)$ of $\operatorname{Aut}(N)$. Sections 3 and 4 are devoted to the reconstruction of primitive elements of $N$ in the group $\Gamma=\operatorname{Aut}(N)$, the key result here says that the stabilizer

$$
\Gamma_{(x)}=\{\sigma \in \operatorname{Aut}(N): \sigma(x)=x\}
$$

where $x$ is a primitive element of $N$ is definable in $\operatorname{Aut}(N)$ with a certain parameter $\pi \in \operatorname{Aut}(N)$. In the final Section 5 we show that it is possible to reconstruct in 
$\operatorname{Aut}(N)$ the multiplication of "independent" primitive elements. This enables us to show that the automorphism $\Delta_{1}$ above can be followed by an inner automorphism of $\operatorname{Aut}(N)$ so that the resulting automorphism $\Delta_{2}$ fixes pointwise some generating set of the group $\operatorname{Aut}(N)$.

\section{Stabilizing COnJugations}

Throughout the paper, $N$ will denote an infinitely generated free nilpotent group and $c$ will always denote nilpotency class of $N$. We shall assume, if not otherwise stated, that $c \geqslant 2$.

The aim of this section is to establish definability of the $\operatorname{subgroup} \operatorname{Inn}(N)$ of all inner automorphisms of $N$ in the group $\operatorname{Aut}(N)$. For convenience's sake, we shall simply call inner automorphisms of $N$ conjugations. By the agreement, if $a \in N$ then conjugation $\tau_{a}$ determined by $a$ is the automorphism

$$
\tau_{a}(z)=a z a^{-1} \quad(z \in N) .
$$

We use standard commutator notation: if $a_{1}, \ldots, a_{s}$ are elements of any group, then $\left[a_{1}, a_{2}\right]=a_{1} a_{2} a_{1}^{-1} a_{2}^{-1}$ and $\left[a_{1}, \ldots, a_{s}\right]=\left[\left[a_{1}, \ldots, a_{s-1}\right], a_{s}\right]$ where $s \geqslant 3$. Given a group $G$, we denote by $\gamma_{k}(G)$ the $k$-th term of the lower central series of $G: \gamma_{1}(G)=G$ and $\gamma_{k+1}(G)=\left[\gamma_{k}(G), G\right](k \in \mathbf{N})$. We denote the group $\gamma_{k}(N)$ by $N_{k}$. Since $N \cong F / \gamma_{c+1}(F)$ where $F$ is a free group having the same rank as $N$, the quotient group $N / N_{k+1}$ where $1 \leqslant k \leqslant c-1$ is a free nilpotent group of nilpotency class $k$; if $\varphi_{k}$ is the homomorphism $\operatorname{Aut}(N) \rightarrow \operatorname{Aut}\left(N / N_{k+1}\right)$, determined by the natural homomorphism $N \rightarrow N / N_{k+1}$, then the kernel of $\varphi_{k}$ will be denoted by $\mathrm{IA}_{k}(N)$. Recall that the elements of the group $\operatorname{IA}_{1}(N)$ are called IA-automorphisms of $N$ and the standard notation for the group $\operatorname{IA}_{1}(N)$ is $\operatorname{IA}(N)$.

In the following proposition we collect some well-known facts about $N$ (its automorphism group $\operatorname{Aut}(N))$ that we shall frequently use below.

Proposition 1.1. (i) Given any basis $\mathcal{X}$ of $N$ and any family $\left\{t_{x}: x \in \mathcal{X}\right\}$ of elements of $N_{k}$ where $k \geqslant 2$, there is an automorphism $\alpha \in \mathrm{IA}_{k-1}(N)$ that takes $x$ to $x t_{x}$ for every $x \in \mathcal{X}$;

(ii) the $c$-th term $N_{c}$ of the lower central series of $N$, a free abelian group, is the centre of the group $N$;

(iii) any IA-automorphism of $N$ fixes each element of $N_{c}$ and any element of $\mathrm{IA}_{c-1}(N)$ fixes each element of $N^{\prime}$, the commutator subgroup of $N$;

(iv) the group $\mathrm{IA}_{c-1}(N)$ is the centre of the group $\mathrm{IA}(N)$, and then $\mathrm{IA}_{c-1}(N)$ is a (torsion-free) abelian group;

(v) the homomorphism $\operatorname{Aut}(N) \rightarrow \operatorname{Aut}\left(N / N_{k+1}\right)$ where $1 \leqslant k \leqslant c-1$, determined by the natural homomorphism $N \rightarrow N / N_{k+1}$, is surjective.

Proof. (i) See Theorem 31.25 in [16.

(ii) See Theorems 31.61, 31.63 of [16.

(iii) Both statements immediately follow from Theorem 5.3 of [15].

(iv) Let $\alpha$ be an arbitrary IA-automorphism and $\eta$ an arbitrary element of $\mathrm{IA}_{c-1}(N)$. Take a basis $\mathcal{X}$ of $N$. Then $\alpha x=x t_{x}$ where $t_{x} \in N^{\prime}$ and $\eta x=x s_{x}$ where $s_{x} \in N_{c}(x \in \mathcal{X})$. By (iii), for all $x \in \mathcal{X}$ we have that $\eta^{-1} x=x s_{x}^{-1}$. Again by (iii),

$$
\eta \alpha \eta^{-1} x=\eta \alpha\left(x s_{x}^{-1}\right)=\eta\left(x t_{x} s_{x}^{-1}\right)=x t_{x}=\alpha x
$$

for every $x \in \mathcal{X}$. 
Conversely, suppose that $\sigma$ is an element of the centre of IA $(N)$. Then for every conjugation $\tau_{a} \in \operatorname{Inn}(N) \subseteq \operatorname{IA}(N)$ we have that

$$
\tau_{a}=\sigma \tau_{a} \sigma^{-1}=\tau_{\sigma(a)}
$$

whence, by (ii), $\sigma a \equiv a\left(\bmod N_{c}\right)$, or, in other words, $\sigma$ is an element of $\operatorname{IA}_{c-1}(N)$.

(v) By (i), any basis of the free nilpotent group $N / N_{k+1}$ can be lifted to a basis of $N$, whence the result.

Proposition 1.2. The group $\operatorname{Aut}(N)$ is centreless.

Proof. We shall use the following claim in due course below; it can be applied in the proof of the Proposition as well.

Claim 1.3. Let $\mathcal{X}$ be any basis of $N$ and $\pi$ be an automorphism of $N$ such that $\pi$ preserves $\mathcal{X}$, fixes $x$ and all $\pi$-orbits on $\mathcal{Y}=\mathcal{X} \backslash\{x\}$ are infinite. Suppose $\alpha$ is an IA-automorphism that commutes with $\pi$. Then $\alpha x=x$.

Proof. Write $\alpha x$ as $x w(x, \bar{y})$ where $w$ is a reduced word over $\mathcal{X}, w(x, \bar{y}) \in N^{\prime}$, and $\bar{y}$ a finite tuple of elements of $\mathcal{Y}$.

Then the condition $\pi \alpha \pi^{-1} x=\alpha x$ implies that

$$
w(x, \bar{y})=\pi^{k} w(x, \bar{y})=w\left(x, \pi^{k} \bar{y}\right) .
$$

for all $k \in \mathbf{Z}$. Since evidently there is a power $\pi^{k}$ of $\pi$ with $\bar{y} \cap \pi^{k} \bar{y}=\varnothing$, then the word $w$ must be trivial.

Now take any basis $\mathcal{X}$ of $N$ and choose for every $x \in \mathcal{X}$ an automorphism $\pi_{x}$ of $N$ such that $\pi_{x}(\mathcal{X})=\mathcal{X}$ and all $\pi_{x}$-orbits on $\mathcal{X}$ but the orbit of $x$ which is equal to $\{x\}$ are infinite.

Suppose $\sigma$ is a central element of $\operatorname{Aut}(N)$. By Proposition1.1(iv), $\sigma \in \operatorname{IA}_{c-1}(N)$. As $\sigma$ commutes with every $\pi_{x}$, we have by Claim 1.3 that $\sigma x=x$ for all $x \in \mathcal{X}$.

Theorem 1.4. The subgroup $\operatorname{Inn}(N)$ of all inner automorphisms of $N$ is a definable subgroup of the group $\operatorname{Aut}(N)$.

Proof. We use induction on nilpotency class $c$ of $N$. When $c=2$, the subgroup $\operatorname{Inn}(N)$ is first-order definable in the group $\operatorname{Aut}(N)$ by Corollary 3.2 of [18].

The key tool for the induction step is provided by the following result from [19]. Let $\widehat{N}$ denote the free nilpotent group $N / N_{c}$ of nilpotency class $c-1$.

Theorem 1.5. 19, Prop. 2.6] The group $\mathrm{IA}_{c-1}(N)$, the kernel of the homomorphism $^{\wedge}: \operatorname{Aut}(N) \rightarrow \operatorname{Aut}(\widehat{N})$, determined by the natural homomorphism $N \rightarrow \widehat{N}$, is first-order definable in the group $\operatorname{Aut}(N)$.

Corollary 1.6. Let $c \geqslant 3$. Suppose that $\operatorname{Inn}(\widehat{N})$ is a definable subgroup of $\operatorname{Aut}(\widehat{N})$. Then any term $\gamma_{k}(\operatorname{Inn}(N))$ where $k \geqslant 2$ of the lower central series of $\operatorname{Inn}(N)$ is definable in the group $\operatorname{Aut}(N)$.

Proof. By Theorem 1.5 the preimage, say $L$ of the group $\operatorname{Inn}(\widehat{N})$ under ${ }^{\wedge}$ is definable in the group $\operatorname{Aut}(N)$. Now $L=\operatorname{Inn}(N) \cdot \operatorname{IA}_{c-1}(N)$. Since the elements of $\operatorname{IA}_{c-1}(N)$ commute with all IA-automorphisms (Proposition 1.1), the commutator subgroup $[L, L]$ of $L$ coincides with that one of $\operatorname{Inn}(N)$ :

$$
[L, L]=[\operatorname{Inn}(N), \operatorname{Inn}(N)] .
$$

We then apply induction on $k$. 
Recall that a primitive element of a relatively free group $G$ is one that can be included in some basis of $G$. We write $\mathrm{NC}(\sigma)$ for the normal closure of a $\sigma \in \operatorname{Aut}(N)$ in the group $\operatorname{Aut}(N)$.

Lemma 1.7. Let $c \geqslant 3$. An automorphism $\sigma$ of $N$ is conjugation by a primitive element of $N$ if and only if

(a) $\widehat{\sigma}$ is conjugation by a primitive element of $\widehat{N}$;

(b) the subgroup $\mathrm{NC}(\sigma)$ contains no elements of the set $\mathrm{IA}_{c-1}(N) \backslash \gamma_{c-1}(\operatorname{Inn}(N))$.

Proof. The necessity part is trivial. Let us prove the converse. An argument similar to one used in the proof of Corollary [1.6] shows that $\gamma_{c-1}(\operatorname{Inn}(N))$ is contained in $\mathrm{NC}(\sigma)$. Take an automorphism $\sigma$ that satisfies the conditions (a) and (b). Then $\sigma$ has the form $\sigma=\tau_{x} \gamma$ for some primitive $x \in N$ and some automorphism $\gamma$ in $\mathrm{IA}_{c-1}(N)$. Suppose, towards a contradiction, that $\sigma$ is not conjugation. Hence $\gamma \notin \gamma_{c-1}(\operatorname{Inn}(N))$.

Observe that if $a$ is a primitive element of $N$ and $\tau_{a} \gamma_{1}$ and $\tau_{a} \gamma_{2}$ where $\gamma_{k} \in$ $\operatorname{IA}_{c-1}(N)(k=1,2)$ are both in $\operatorname{NC}(\sigma)$, then $\gamma_{1} \equiv \gamma_{2}\left(\bmod \gamma_{c-1}(\operatorname{Inn}(N))\right)$; indeed, otherwise the element $\left(\tau_{a} \gamma_{1}\right)\left(\tau_{a} \gamma_{2}\right)^{-1}$ is a member of $\operatorname{IA}_{c-1}(N) \backslash \gamma_{c-1}(\operatorname{Inn}(N))$, contradicting (b).

Let $\mathcal{X}$ be a basis of $N$ which contains $x$. Write $\mathcal{Y}$ for $\mathcal{X} \backslash\{x\}$. Suppose that $\gamma x=x w(x, \bar{y})$ where $w$ is a reduced word over $\mathcal{X}$ and $\bar{y}$ a finite tuple of elements of $\mathcal{Y}$. Consider an automorphism $\pi$ of $N$ which preserves $\mathcal{X}$, fixes $x$ and such that tuples $\pi \bar{y}$ and $\bar{y}$ have no common elements. As $\pi x=x$ we have by the observation above that $\gamma^{\pi}=\gamma \tau_{s}$ where $s \in N_{c-1}$. It follows that

$$
x w(x, \pi \bar{y})=s x s^{-1} w(x, \bar{y}) .
$$

Consider an endomorphism $\varepsilon$ of $N$ which takes all elements of $\pi \bar{y}$ to 1 and fixes all other elements of $\mathcal{X}$. By applying $\varepsilon$ to the both parts of (1), we get that $w(x, \bar{y})=$ $t x^{-1} t^{-1} x$ where $t=\varepsilon(s)$. As $t x^{-1} t^{-1} x=\left[t, x^{-1}\right]=\left[t^{-1}, x\right]$ is in the centre of $N$, we have that

$$
\gamma x=x w(x, \bar{y})=x\left(t^{-1} x t x^{-1}\right)=\left(t^{-1} x t x^{-1}\right) x=t^{-1} x t .
$$

Hence the automorphism $\tau_{t} \gamma$ fixes $x$. Now $\sigma=\tau_{x} \gamma=\tau_{x t^{-1}} \tau_{t} \gamma$, and conjugating $\sigma$ by any IA-automorphism that sends $x t^{-1}$ to $x$, we obtain that $\tau_{x}\left(\tau_{t} \gamma\right)$ is $\operatorname{in} \mathrm{NC}(\sigma)$. Thus, without loss of generality, we may assume that $\gamma x=x$.

It is easy to see that $x$ (any primitive element of $N$ ) commutes with an element $s \in N_{c-1}$ if and only if $s \in N_{c}$, the centre of $N$. Let $\rho$ be any automorphism of $N$ which takes $x$ to itself. Again, $\gamma^{\rho}=\gamma \tau_{s}$ for a suitable $s \in N_{c-1}$, whence $x=s x s^{-1}$, and then $s \in N_{c}$. Therefore $\tau_{s}=$ id, or, in other words, $\gamma$ commutes with $\rho$.

Consider an arbitrary element $y \in \mathcal{Y}$. Assume that $\gamma y=y w_{y}(y, x, \bar{z})$ where $w_{y}$ is a reduced word over $\mathcal{X}$ and $\bar{z}$ is a finite tuple of elements of $\mathcal{X} \backslash\{x, y\}$. As it is easy to find an automorphism $\pi$ of $N$, preserving $\mathcal{X}$, fixing both $x$ and $y$ (and hence commuting with $\gamma$ ), and such that $\pi \bar{z} \cap \bar{z}=\varnothing$, the tuple $\bar{z}$ must be empty. Further, by considering an automorphism interchanging distinct elements $y_{1}, y_{2} \in \mathcal{Y}$ and fixing all elements in $\mathcal{X} \backslash\left\{y_{1}, y_{2}\right\}$, we see that there is a group word $w\left(*_{1}, *_{2}\right)$ over an alphabet having empty intersection with $N$ such that $w(y, x)=$ $\left[w\left(*_{1}, *_{2}\right)\right]_{y}^{*_{1}, *_{2}}=w_{y}(y, x)$ for every $y \in \mathcal{Y}$ (or, somewhat more formally, there is a term $w\left(*_{1}, *_{2}\right)$ in two variables of the language of group theory such that its value $w(y, x)$ at $(y, x)$ is $w_{y}$ for every $\left.y \in \mathcal{Y}\right)$. 
Also, taking distinct $y, z \in \mathcal{Y}$ and considering the automorphism $\rho$ of $N$ with $\rho y=y z$, fixing $\mathcal{X} \backslash\{y\}$ pointwise, we obtain that

$$
w(y z, x)=w(y, x) w(z, x) .
$$

Fix a $y \in \mathcal{Y}$. Consider the automorphism $\pi$ of $N$ interchanging $x$ and $y$ and fixing pointwise $\mathcal{X} \backslash\{x, y\}$, the automorphism $\rho$ which takes $x$ to $x y$ and fixes $\mathcal{Y}$ pointwise and any IA-automorphism $\alpha$ taking $x y x^{-1}$ to $y$. We then have that the automorphism

$$
\left[\left(\tau_{x} \gamma\right)^{\rho}\left(\tau_{x} \gamma\right)^{-1}\right]^{\alpha}=\left[\tau_{x y x^{-1}} \gamma^{\rho} \gamma^{-1}\right]^{\alpha}=\tau_{y} \gamma^{\rho} \gamma^{-1}
$$

belongs to $\mathrm{NC}(\sigma)$, and so does the automorphism $\tau_{y} \gamma^{\rho}=\left(\tau_{x} \gamma\right)^{\pi}$. Therefore for some $s \in N_{c-1}$

$$
\gamma^{\rho} \gamma^{-1}=\tau_{s} \gamma^{\pi}
$$

One readily verifies that $(3)$ implies that

$$
z w(z, x y)=s z s^{-1} w(z, x) w(z, y)
$$

for every $z \in \mathcal{Y}$. In particular,

$$
y w(y, x y)=\operatorname{sys}^{-1} w(y, x) .
$$

Let $\varepsilon$ be the endomorphism of $N$ which sends $x$ to $y$ and fixes $\mathcal{Y}$ pointwise. Then $y=\varepsilon(s) y \varepsilon(s)^{-1}$, and hence $\varepsilon(s) \in N_{c}$. Fix a $z \in \mathcal{Y} \backslash\{y\}$ and apply $\varepsilon$ to the both parts of (4), thereby getting that

$$
z w\left(z, y^{2}\right)=z w(z, y)^{2} \text {, or } w\left(z, y^{2}\right)=w(z, y)^{2} .
$$

By (4), we also have that $w\left(z^{2}, y\right)=w(z, y)^{2}$. We then claim that $w(z, y)=1$. We define a function $\nu_{z}$ on the set of all basic commutators over $\{z, y\}$ taking values in $\mathbf{N}: \nu_{z}(z)=1, \nu_{z}(y)=0$ and $\nu_{z}\left(\left[c_{1}, c_{2}\right]\right)=\nu_{z}\left(c_{1}\right)+\nu_{z}\left(c_{2}\right)$ where $c_{1}, c_{2}$ are basic commutators over $\{z, y\}$. The function $\nu_{y}$ is defined similarly. Let $b=b(z, y)$ be a basic commutator of weight $c$ over $\{z, y\}$. Observe that $\nu_{z}(b)+\nu_{y}(b)=c$ and

$$
b\left(z^{k}, y\right) b\left(z, y^{k}\right)=b(z, y)^{k^{\nu_{z}(b)}} b(z, y)^{k^{\nu_{z}(b)}}=b(z, y)^{k^{c}}
$$

where $k$ is a natural number. Therefore

$$
v\left(z^{k}, y\right) v\left(z, y^{k}\right)=v(z, y)^{k^{c}} \quad(k \in \mathbf{N})
$$

for every element/word $v \in\langle z, y\rangle \cap N_{c}$. It then follows that

$$
w(z, y)^{4}=w(z, y)^{2} w(z, y)^{2}=w\left(z^{2}, y\right) w\left(z, y^{2}\right)=w(z, y)^{2^{c}}
$$

As $4<2^{c}, w(z, y)=1$. Then $\gamma=\mathrm{id}$, which completes the proof of the lemma.

As it was said above, the $\operatorname{group} \operatorname{Inn}(N)$ is definable in $\operatorname{Aut}(N)$ when $c=2$; then so is the family of all conjugations by primitive elements, for $\operatorname{Inn}(N)=\mathrm{NC}(\tau)$ where $\tau \in \operatorname{Inn}(N)$ if and only if $\tau$ is conjugation by a primitive element. Then by Lemma 1.7 conjugations by primitive elements are definable in the group $\operatorname{Aut}(N)$ for any $c \geqslant 2$, and hence the subgroup $\operatorname{Inn}(N)$ is definable in the group $\operatorname{Aut}(N)$ for any $c \geqslant 2$.

Now we obtain some corollaries of Theorem 1.4

Corollary 1.8. All subgroups $\mathrm{IA}_{k}(N)$ where $1 \leqslant k \leqslant c-1$ are definable in the group $\operatorname{Aut}(N)$.

Proof. By Theorem 1.5 and by Theorem 1.4. 
Proposition 1.9. (i) Let $\Delta \in \operatorname{Aut}(\operatorname{Aut}(N))$. Then there is an inner automorphism $T_{\pi} \in \operatorname{Aut}(\operatorname{Aut}(N))$ such that the composition $T_{\pi} \circ \Delta$ fixes the subgroup $\operatorname{Inn}(N)$ pointwise;

(ii) suppose that $\Delta \in \operatorname{Aut}(\operatorname{Aut}(N))$ fixes every element of $\operatorname{Inn}(N)$. Then

$$
\Delta \sigma \equiv \sigma\left(\bmod \mathrm{IA}_{c-1}(N)\right)
$$

for all $\sigma \in \operatorname{Aut}(N)$.

Proof. (i) We saw above that conjugations by primitive elements of $N$ are definable; a generating set $X$ of $\operatorname{Inn}(N)$ consisting of conjugations by primitive elements is a basis of $\operatorname{Inn}(N)$ if and only if

$$
\tau \notin\langle X \backslash\{\tau\}\rangle \quad \forall \tau \in X
$$

where $\langle X \backslash\{\tau\}\rangle$ is the subgroup of $\operatorname{Aut}(N)$ generated by the set $X \backslash\{\tau\}$. Thus every automorphism of the group $\operatorname{Aut}(N)$ takes an arbitrary basis of $\operatorname{Inn}(N)$ to another basis of $\operatorname{Inn}(N)$.

Fix a basis $X$ of $\operatorname{Inn}(N)$ and a $\Delta \in \operatorname{Aut}(\operatorname{Aut}(N))$. As $\Delta(X)$ is a basis of $\operatorname{Inn}(N)$, there is a $\rho \in \operatorname{Aut}(N)$ such that

$$
\Delta(\tau)=\rho \tau \rho^{-1} \quad(\tau \in X) .
$$

Now if $T_{\rho^{-1}}$ is the inner automorphism of the group $\operatorname{Aut}(N)$ determined by $\rho^{-1}$, that is, if

$$
T_{\rho^{-1}}(\sigma)=\rho^{-1} \sigma \rho \quad(\sigma \in \operatorname{Aut}(N)),
$$

then $T_{\rho^{-1}} \circ \Delta$ fixes $X$ pointwise.

(ii). Let $z$ be any element of $N$ and $\sigma \in \operatorname{Aut}(N)$. We have that

$$
\tau_{\sigma z}=\Delta\left(\tau_{\sigma z}\right)=\Delta\left(\sigma \tau_{z} \sigma^{-1}\right)=\Delta(\sigma) \tau_{z} \Delta(\sigma)^{-1}=\tau_{\Delta(\sigma) z} .
$$

Then $\sigma z \equiv \Delta(\sigma) z\left(\bmod N_{c}\right)$ for every $z \in N$, and hence $\sigma^{-1} \Delta(\sigma) \in \operatorname{IA}_{c-1}(N)$.

\section{Stabilizing IA-AUtomorphisms}

Let $\mathcal{X}$ be any basis of $N$. Then by $\operatorname{Sym}(\mathcal{X})$ (as it is, for instance, done in [5]) we denote the group of all automorphisms of $N$ that preserve $\mathcal{X}$ setwise. For simplicity's sake, we call elements of $\operatorname{Sym}(\mathcal{X})$ permutational automorphisms relative to $\mathcal{X}$. Similarly, if $X$ is a basis of the group $\operatorname{Inn}(N)$ (a basis set of conjugations), the symbol $\operatorname{Sym}(X)$ denotes the group of all automorphisms of $N$ acting on $X$ as permutations.

Remark 2.1. Suppose that the elements of a basis $\mathcal{X}$ of $N$ determine the elements of a given basis set of conjugations $X: X=\left\{\tau_{x}: x \in \mathcal{X}\right\}$ (in similar situations below we shall also say that a basis of $N$ determines a given basis of $\operatorname{Inn}(N)$ ). Then for any $\rho \in \operatorname{Sym}(X)$ there is a uniquely determined $\sigma \in \operatorname{Sym}(\mathcal{X})$ such that the actions of $\rho$ and $\sigma$ on $\operatorname{IA}(N)$ are the same:

$$
\rho \alpha \rho^{-1}=\sigma \alpha \sigma^{-1}
$$

for every $\alpha \in \operatorname{IA}(N)$. Indeed, it follows from the assumptions made that there is an $\eta \in \mathrm{IA}_{c-1}(N)$ with $\rho=\sigma \eta$. Then (6) is a corollary of the fact that elements of $\operatorname{IA}_{c-1}(N)$ commute with all elements of $\operatorname{IA}(N)$ (Proposition 1.1).

In particular, every time when we need an element of $\operatorname{Sym}(X)$ that acts on $\operatorname{IA}(N)$, we can use a suitable element of $\operatorname{Sym}(\mathcal{X})$ and vice versa. 
We use below the notation from the theory of permutation groups. Recall that given a group $G$ acting on a set $A$ we denote by $G_{(B)}$ and $G_{\{B\}}$ the pointwise and the setwise stabilizers of a subset $B$ of $A$, respectively. Any symbol of the form $G_{*_{1}, *_{2}}$ is the intersection of subgroups $G_{*_{1}}$ and $G_{*_{2}}$. It is convenient, when working with subgroups $G$ of $\operatorname{Aut}(N)$ and when using some basis $\mathcal{B}$ of $N$, to denote simply by $G_{\langle\mathcal{C}\rangle}$ where $\mathcal{C} \subseteq \mathcal{B}$ the subgroup $G_{(\mathcal{B} \backslash \mathcal{C}),\{\langle\mathcal{C}\rangle\}}$ (consisting of elements of $G$ that fix the set $\mathcal{B} \backslash \mathcal{C}$ pointwise and preserve the subgroup of $N$ generated by $\mathcal{C}$ ).

Proposition 2.2. Let $x$ be a primitive element of $N$ and let $k$ with $1 \leqslant k \leqslant c-1$ be a natural number. Then the group $\operatorname{IA}_{k}(N)_{(x)}$, consisting of the elements of the group $\mathrm{IA}_{k}(N)$ stabilizing $x$, is definable with the parameter $\tau_{x}$ in the group $\operatorname{Aut}(N)$

Proof. Let $X$ be any basis set of conjugation of which $\tau_{x}$ forms a part. Write $Y$ for $X \backslash\left\{\tau_{x}\right\}$. Choose also a basis $\mathcal{X}$ of $N$ such that the elements of $\mathcal{X}$ determine the elements of $X$; we assume that $x \in \mathcal{X}$.

Consider an element $\pi^{*}$ of $\operatorname{Sym}(X)$ which, under the conjugation action, fixes $\tau_{x}$ and moves elements of $Y$ as a permutation having no fixed points and having infinitely many infinite orbits. According to Remark 2.1 we may assume without loss of generality (for we are going to consider conjugation actions of elements of $\operatorname{Aut}(N)$ on $\operatorname{IA}(N))$ that $\pi^{*} x=x$ and that infinitely many orbits of the action of $\pi^{*}$ on $\mathcal{Y}=\mathcal{X} \backslash\{x\}$ are all infinite.

By Claim 1.3, if an $\eta \in \mathrm{IA}_{c-1}(N)$ commutes with $\pi^{*}$ (with any element of the coset $\left.\pi^{*} \mathrm{IA}_{c-1}(N)\right)$, then $\eta x=x$.

When using symbols like $G_{(*)}$ with $G=\operatorname{Sym}(\mathcal{X})$ or $G=\operatorname{Sym}(X)$, we shall replace $\operatorname{Sym}(\mathcal{X})$ and $\operatorname{Sym}(X)$ by $\Pi$ and $P$, respectively. By $Z^{+}\left(\pi^{*}\right)$ we will denote the subgroup of the centralizer $Z\left(\pi^{*}\right)$ of $\pi^{*}$ in the group Aut $(N)$ consisting of automorphisms sending $x$ to itself.

Let $L$ be a free nilpotent group, $\mathcal{B}$ a basis of $L$ and $\mathcal{B}=\bigcup_{i \in I} \mathcal{B}_{i}$ be a partition of $\mathcal{B}$. If $\rho_{i}$ is an automorphism of the subgroup $\left\langle\mathcal{B}_{i}\right\rangle(i \in I)$ we shall denote by $\circledast_{i \in I} \rho_{i}$ the only automorphism of $L$ which extends all automorphisms $\rho_{i}$.

The following result will be used in Section 4 as well. Recall that a moiety of a given infinite set $I$ is any subset $J$ of $I$ with $|J|=|I \backslash J|$.

Lemma 2.3. Let $\mathcal{Y}_{0} \subseteq \mathcal{Y}$ be a subset of $\mathcal{Y}$ maximal with the property "there are no elements lying in the same orbit of $\pi^{*} "$ and let $\mathcal{Z}_{0}$ be a moiety of $\mathcal{Y}_{0}$. Take an arbitrary automorphism $\gamma$ of the group $\left\langle x, \mathcal{Z}_{0}\right\rangle$ that fix $x$. Then there is a $\sigma \in Z^{+}\left(\pi^{*}\right)$ and $a \rho \in \Pi_{(x)}=\operatorname{Sym}(\mathcal{X})_{(x)}$ such that the automorphism $\sigma \sigma^{\rho}$ fixes all elements of $\mathcal{X} \backslash \mathcal{Z}_{0}$ and coincides with $\gamma$ on $\mathcal{Z}_{0}$. In the case when $\gamma \in \operatorname{IA}_{k}\left(\left\langle x, \mathcal{Z}_{0}\right\rangle\right), \sigma$ can be taken from $\mathrm{IA}_{k}(N)$.

Proof. Write $\mathcal{Y}_{k}$ for $\left(\pi^{*}\right)^{k} \mathcal{Y}_{0}$ where $k \in \mathbf{Z}$. Hence

$$
\mathcal{Y}=\bigcup_{k \in \mathbf{Z}} \mathcal{Y}_{k}
$$

is a partition of $\mathcal{Y}$ into moieties.

We then partition $\mathcal{Y}_{0} \backslash \mathcal{Z}_{0}$ into countably many moieties indexed by elements of $\mathbf{N} \backslash\{0\}$, getting that

$$
\mathcal{Y}_{0}=\bigcup_{n \in \mathbf{N}} \mathcal{Z}_{n}
$$

Assume that $\mathcal{Z}_{k}=\left\{z_{i, k}: i \in I\right\}$ where $k$ runs over $\mathbf{N}$. 
First, to illustrate the idea we consider the case when $\gamma$ preserves the group $\left\langle\mathcal{Z}_{0}\right\rangle$; let $\nu$ denote the restriction of $\gamma$ on $\left\langle\mathcal{Z}_{0}\right\rangle$. Our goal is then to construct an automorphism $\sigma_{0}$ of $\left\langle\mathcal{Y}_{0}\right\rangle$ that can be written as a $\circledast$-product of automorphisms of the groups $\left\langle\mathcal{Z}_{k}\right\rangle$ of the form

$$
\underbrace{\nu \circledast \nu^{-1} \circledast \mathrm{id}} \circledast \underbrace{\nu \circledast \nu^{-1} \circledast \mathrm{id}} \circledast \ldots \circledast \underbrace{\nu * \nu^{-1} \circledast \mathrm{id}} \circledast \ldots
$$

where the symbols $\nu, \nu^{-1}$ represent the action (up to an isomorphism of actions) of $\sigma_{0}$ on subgroups $\left\langle\mathcal{Z}_{k}\right\rangle(k \in \mathbf{N})$. Formally, for every $k \in \mathbf{Z}$ we denote the bijection

$$
z_{i, 0} \mapsto z_{i, k} \quad(i \in I)
$$

from $\mathcal{Z}_{0}$ to $\mathcal{Z}_{k}$ by $\lambda_{k}$ and then extend $\lambda_{k}$ to the isomorphism $\lambda_{k}^{*}$ of groups $\left\langle\mathcal{Z}_{0}\right\rangle$ and $\left\langle\mathcal{Z}_{k}\right\rangle$. Then $\sigma_{0}$ is defined as follows:

$$
\begin{array}{ll}
\sigma_{0}\left(z_{i, k}\right)=\lambda_{k}^{*} \gamma\left(z_{i, 0}\right), & \text { if } k \equiv 0(\bmod 3) \\
\sigma_{0}\left(z_{i, k}\right)=\lambda_{k}^{*} \gamma^{-1}\left(z_{i, 0}\right), & \text { if } k \equiv 1(\bmod 3) \\
\sigma_{0}\left(z_{i, k}\right)=z_{i, k} & \text { if } k \equiv 2(\bmod 3)
\end{array}
$$

for every $i \in I$.

Using bijections $\lambda_{k}$, one can construct permutational automorphisms $\rho_{0}$ and $\rho_{1}$ of the group $\left\langle\mathcal{Y}_{0}\right\rangle$ such that the conjugates $\sigma_{0}^{\rho_{0}}$ and $\sigma_{0}^{\rho_{1}}$ are automorphisms of the form

and of the form

$$
\underbrace{\nu^{-1} \circledast \nu \circledast \mathrm{id}} \circledast \underbrace{\nu^{-1} \circledast \nu \circledast \mathrm{id}} \circledast \ldots \circledast \underbrace{\nu^{-1} \circledast \nu \circledast \mathrm{id}} \circledast \ldots
$$

$$
\mathrm{id} \circledast \nu \circledast \mathrm{id} \circledast \underbrace{\nu^{-1} \circledast \nu \circledast \mathrm{id}} \circledast \ldots \circledast \underbrace{\nu^{-1} \circledast \nu \circledast \mathrm{id}} \circledast \ldots
$$

respectively, and the products $\sigma_{0}^{\rho_{0}} \sigma_{0}$ and $\sigma_{0}^{\rho_{1}} \sigma_{0}$ are the identity automorphism and the automorphism

$$
\nu \circledast \mathrm{id} \circledast \mathrm{id} \circledast \ldots \circledast \mathrm{id} \circledast \mathrm{id} \circledast \mathrm{id} \circledast \ldots
$$

of $\left\langle\mathcal{Y}_{0}\right\rangle$, respectively.

It is then easy to extend $\sigma_{0}$ to the sets $\mathcal{Y}_{k}$ with $k \neq 0$ aiming at obtaining an element $\sigma$ of $Z^{+}\left(\pi^{*}\right)$ that fixes $x$ and commutes with $\pi^{*}$.

There is a permutational automorphism $\rho \in \Pi_{(x)}$ such that

- $\rho$ fixes $x$;

- the action of $\rho$ on any set $\mathcal{Y}_{k}$ where $k \neq 0$ is isomorphic to that one of $\rho_{0}$ on $\mathcal{Y}_{0}$;

- the restriction of $\rho$ on $\mathcal{Y}_{0}$ is $\rho_{1}$;

- the product $\sigma \sigma^{\rho}$ acts trivially on any $\mathcal{Y}_{k}$ where $k \neq 0$ and the restriction of $\rho$ on $\left\langle\mathcal{Y}_{0}\right\rangle$ equals the automorphism (8).

In particular, $\sigma \sigma^{\rho} \in \Gamma_{\left(\mathcal{X} \backslash \mathcal{Z}_{0}\right),\left\{\left\langle\mathcal{Z}_{0}\right\rangle\right\}}=\Gamma_{\left\langle\mathcal{Z}_{0}\right\rangle}$ where $\Gamma=\operatorname{Aut}(N)$.

Now it is easy to check that permutational automorphisms $\rho_{0}, \rho_{1}$ relative to $\mathcal{Y}_{0}$ and a permutational automorphism $\rho \in \Pi_{(x)}$ can also be found in the general case when the images $\gamma\left(z_{i, 0}\right) \in\left\langle x, \mathcal{Z}_{0}\right\rangle$ of elements of $\mathcal{Z}_{0}$ are not necessarily in $\left\langle\mathcal{Z}_{0}\right\rangle$.

Assume some basis $\mathcal{U}$ of $N_{c}$ consisting of basic commutators of weight $c$ over $\mathcal{X}$ is fixed.

Write $K$ for the group $\mathrm{IA}_{c-1}(N)$. Lemma 2.3 implies that there is a moiety $\mathcal{Z}_{0}$ of $\mathcal{Y}$ such that the subgroup $K_{\left\langle\mathcal{Z}_{0}\right\rangle}$ (the notation here and up to the end of the proof is relative to the basis $\mathcal{X}$, if not otherwise stated) and the set, say $B_{x}$ of elements of $K_{\left(\mathcal{X} \backslash \mathcal{Z}_{0}\right)}$ which take $z_{i, 0} \in \mathcal{Z}_{0}$ either to itself, or to an element $z_{i, 0} t_{i}$ where all 
elements of $\mathcal{U}$ participating in the decomposition of $t_{i}$ have occurrences of $x(i \in I)$, are both contained in the set

$$
\left\{\eta \eta^{\rho}: \eta \in \mathrm{IA}_{c-1}(N), \rho \in \Pi_{(x)},\left[\eta, \pi^{*}\right]=\mathrm{id}\right\}
$$

which is equal to the set

$$
\left\{\eta \eta^{\rho}: \eta \in \mathrm{IA}_{c-1}(N), \rho \in P_{\left(\tau_{x}\right)},\left[\eta, \pi^{*}\right]=\mathrm{id}\right\}
$$

and hence definable with the parameters $\tau_{x}, X$ and $\pi^{*}$.

The following statement is essentially a part of the proof of Theorem 2.5 in 20 .

Claim 2.4. Given a moiety $\mathcal{C}$ of a basis $\mathcal{B}$ of $N$, every element of $K$ can be written as a product of at most $2(c+1)$ conjugates of elements of $K_{\langle\mathcal{C}\rangle}$ by permutational automorphisms relative to $\mathcal{B}$.

For the reader's convenience, we reproduce the proof, for we shall need below both the statement and the method of its proof to be applied in somewhat different situations.

Proof. We can write $K$ as $K=K_{(\mathcal{B} \backslash \mathcal{C})} \cdot K_{(\mathcal{C})}$ (Proposition 1.1 (i) and (iii)). It suffices to prove that every element of $K_{(\mathcal{C})}$ is a product of at most $c+1$ conjugates of $K_{\langle\mathcal{C}\rangle}$ by permutational automorphisms, because the groups $K_{(\mathcal{B} \backslash \mathcal{C})}$ and $K_{(\mathcal{C})}$ are conjugate by some permutational automorphism. Write $\mathcal{D}$ for $\mathcal{B} \backslash \mathcal{C}$ and let $\mathcal{D}=\left\{d_{i}: i \in I\right\}$. Take an $\alpha \in K_{(\mathcal{C})}$. Then $\alpha$ acts trivially on $\mathcal{C}$ and

$$
\alpha d_{i}=d_{i} s_{i} \quad(i \in I)
$$

where $s_{i} \in N_{c}$. We make use of the fact that any basic commutator of weight $c$ over $\mathcal{B}$ is formed from at most $c$ elements of $\mathcal{B}$. Thus we write $\mathcal{C}$ as a union of $c+1$ disjoint moieties:

$$
\mathcal{C}=\mathcal{C}_{1} \cup \ldots \cup \mathcal{C}_{c+1}
$$

We write $s_{i}$ as a product of elements of some basis of $N_{c}$, basic commutators over $\mathcal{B}$. We then rewrite $s_{i}(i \in I)$ in the form

$$
s_{i}=s_{i, 1} \ldots s_{i, c+1}
$$

collecting into $s_{i, k}$ all those basis elements in the decomposition of $s_{i}$ that do not contain, as basic commutators, occurrences of letters from $\mathcal{C}_{k}(k=1, \ldots, c+1)$. Then we define $c+1$ elements of $\operatorname{IA}_{c-1}(N)$ such that their product $\alpha_{1} \ldots \alpha_{c+1}$ is equal to $\alpha$ :

$$
\begin{array}{llrl}
\alpha_{k} d_{i} & =d_{i} s_{i, k}, & & (i \in I), \\
\alpha_{k} d & =d, & & (d \in \mathcal{C}) .
\end{array}
$$

As $\alpha_{k} \in K_{\left\langle\mathcal{D} \cup\left(\mathcal{C} \backslash \mathcal{C}_{k}\right)\right\rangle}=K_{\left\langle\mathcal{B} \backslash \mathcal{C}_{k}\right\rangle}(k=1, \ldots, c+1)$, we have the desired, for the subgroups $K_{\left\langle\mathcal{B} \backslash \mathcal{C}_{k}\right\rangle}$ and $K_{\langle\mathcal{C}\rangle}$ are conjugate by a permutational automorphism.

It follows from the Claim that the group $K_{\langle\mathcal{Y}\rangle}$ is contained in the group generated by conjugates of elements of $K_{\left\langle\mathcal{Z}_{0}\right\rangle}$ by elements of $P_{\left(\tau_{x}\right)}$. Similarly, the proof of the Claim can be applied to show that the subgroup generated by conjugates of elements of $B_{x} \cup K_{\left\langle\mathcal{Z}_{0}\right\rangle}$ by elements of $P_{\left(\tau_{x}\right)}$ contains the family $C_{x}$ of all elements $\{\alpha\}$ of $\mathrm{IA}_{c-1}(N)$ such that $\alpha x=x$ and the image any element $y \in \mathcal{Y}$ is of the form $y t_{y}$ where $t_{y} \in N_{c}$ is either 1 , or again every basis element of $\mathcal{U}$ appearing in the decomposition of $t_{y}$ has occurrences of $x$. We complete the proof for the group $K_{(x)}$ by observing that

$$
K_{(x)}=K_{\langle\mathcal{Y}\rangle} \cdot C_{x}
$$


Before going on, let us summarize the proof of definability of $K_{(x)}=\operatorname{IA}_{c-1}(N)_{(x)}$ over $\tau_{x}$ : we have proved that there is a basis set $X$ of conjugations with $\tau_{x} \in X$, an element $\pi^{*}$ of $P=\operatorname{Sym}(X)$ acting on $X$ in the manner described above such that the group $\mathrm{IA}_{c-1}(N)_{(x)}$ equals the subgroup generated by conjugates of elements of $\mathrm{IA}_{c-1}(N)$ commuting with $\pi^{*}$ by elements of $P_{\left(\tau_{x}\right)}$ :

$$
\mathrm{IA}_{c-1}(N)_{(x)}=\left\langle\rho \eta \rho^{-1}: \rho \in P_{\left(\tau_{x}\right)}, \eta \in \mathrm{IA}_{c-1}(N),\left[\eta, \pi^{*}\right]=\mathrm{id}\right\rangle .
$$

Lemma 2.5. For every $k$ with $1 \leqslant k \leqslant c-1$

$$
\operatorname{IA}_{k}(N)_{(x)}=\left\langle\rho \eta \rho^{-1}: \rho \in P_{\left(\tau_{x}\right)}, \eta \in \operatorname{IA}_{k}(N),\left[\eta, \pi^{*}\right]=\mathrm{id}\right\rangle .
$$

Proof. We prove the statement by induction on $k$. Suppose that (9) is true for the group $\operatorname{IA}_{k}(N)$ and $k \geqslant 2$. Write $J$ for the group

$$
\left\langle\rho \eta \rho^{-1}: \rho \in P_{\left(\tau_{x}\right)}, \eta \in \mathrm{IA}_{k-1}(N),\left[\eta, \pi^{*}\right]=\mathrm{id}\right\rangle .
$$

Then $J \subseteq \operatorname{IA}_{k-1}(N)_{(x)}$. It is also clear that $\operatorname{IA}_{k}(N)_{(x)}$ is contained in $J$ by the induction hypothesis.

We consider the natural homomorphism $\sim$ from the group $N$ onto the free nilpotent group $\widetilde{N}=N / N_{k+1}$ of nilpotency class $k$. The homomorphism $\sim$ determines the homomorphism $\operatorname{Aut}(N) \rightarrow \operatorname{Aut}(\widetilde{N})$ which we will denote by the same symbol $\sim$. By the above considerations on the group $\operatorname{IA}_{c-1}(N)$ we have that

$$
\operatorname{IA}_{k-1}(\widetilde{N})_{(\widetilde{x})}=\left\langle\rho \eta \rho^{-1}: \rho \in P_{\left(\widetilde{\tau}_{x}\right)}, \eta \in \operatorname{IA}_{k-1}(\widetilde{N}),\left[\eta, \widetilde{\pi^{*}}\right]=\mathrm{id}\right\rangle
$$

Note that since the homomorphism $\operatorname{Aut}(N) \rightarrow \operatorname{Aut}(\widetilde{N})$ is surjective (Proposition 1.1 (iv)), then

$$
\widetilde{J}=\operatorname{IA}_{k-1}(\tilde{N})_{(\widetilde{x})}
$$

Let $\alpha \in \operatorname{IA}_{k-1}(N)_{(x)}$. As $\alpha x=x$, then $\widetilde{\alpha}(\widetilde{x})=\widetilde{x}$, and by $(10) \widetilde{\alpha} \in \widetilde{J}$. Hence there exist an $\eta \in J$ and a $\beta \in \mathrm{IA}_{k}(N)$ such that $\alpha=\beta \eta$. Suppose that $\beta x=x t$ where $t \in N_{k+1}$. We have that $\alpha x=\beta \eta(x)=\beta(x)=x t$, whence $t=1$. Therefore $\beta \in \mathrm{IA}_{k}(N)_{(x)} \subseteq J$, and we are done.

The proof of Proposition 2.2 is now completed.

We are going to make use of the following result from paper [4] by Bryant and Gupta.

Theorem 2.6. Let $F_{n, c}$ be a free nilpotent group of rank $n \geqslant 3$ and of class $c \geqslant 3$ where $n \geqslant c-1,(c+1) / 2$. Let $x_{1}, \ldots, x_{n}$ be a basis of $F_{n, c}$. Consider the automorphism $\delta_{c} \in \operatorname{Aut}\left(F_{c}\right)$ defined via

$$
\begin{aligned}
\delta_{c}: \quad x_{1} & \mapsto x_{1}\left[x_{1}, x_{2}, \ldots, x_{c-1}, x_{1}\right], \\
x_{i} & \mapsto x_{i}, \quad i>1 .
\end{aligned}
$$

Then the group $I_{c-1}=\mathrm{IA}_{c-1}\left(F_{n, c}\right)$ is generated by conjugates of elements of the set $\left(I^{\prime} \cap I_{c-1}\right) \cup\left\{\delta_{c}\right\}$, where $I=\mathrm{IA}\left(F_{n, c}\right)$ and $I^{\prime}$ is the commutator subgroup of $I$, by tame automorphisms of $F_{n, c}$.

(See [4, p. 313, p. 317] for the details; for applications below, it will be enough to know that $\mathrm{IA}_{c-1}\left(F_{n, c}\right)$ is contained in the normal closure of the set $\left.\left(I^{\prime} \cap I_{c-1}\right) \cup\left\{\delta_{c}\right\}\right)$.

In the first part of the proof of our next result, we shall check that any automorphism $\Delta$ of the group $\operatorname{Aut}(N)$ stabilizing all conjugations, stabilizes an analogue of $\delta_{c}$ in the group $\operatorname{Aut}(N)$. 
Proposition 2.7. Let $\Delta$ be an automorphism of the group $\operatorname{Aut}(N)$ stabilizing all elements of the group $\operatorname{Inn}(N)$. Then $\Delta$ fixes the group $\mathrm{IA}_{2}(N)$ elementwise.

Proof. First, we observe that any automorphism $\Delta$ satisfying the conditions of the Proposition takes any commutator of elements of $\operatorname{IA}(N)$, and hence any element of the commutator subgroup of $\operatorname{IA}(N)$, to itself. Indeed, let $\alpha_{1}, \alpha_{2} \in \operatorname{IA}(N)$. By Proposition 1.9 (ii), $\Delta\left(\alpha_{k}\right)=\alpha_{k} \eta_{k}$ where $\eta_{k} \in \mathrm{IA}_{c-1}(N), k=1$, 2. Therefore

$$
\Delta\left(\alpha_{1} \alpha_{2} \alpha_{1}^{-1} \alpha_{2}^{-1}\right)=\alpha_{1} \eta_{1} \cdot \alpha_{2} \eta_{2} \cdot \eta_{1}^{-1} \alpha_{1}^{-1} \cdot \eta_{2}^{-1} \alpha_{2}^{-1}=\alpha_{1} \alpha_{2} \alpha_{1}^{-1} \alpha_{2}^{-1},
$$

since $\eta_{1}, \eta_{2}$ are central elements of the group $\operatorname{IA}(N)$. In a similar vein, one proves that if $\alpha \in \operatorname{IA}(N)$ is stabilized by $\Delta$, then so is any conjugate of $\alpha$ by an element of $\operatorname{Aut}(N)$.

Consider a basis $\mathcal{B}$ of $N$ and write it in the form

$$
\mathcal{B}=\left\{x_{k}: k \in \mathbf{N}, k \geqslant 1\right\} \cup \mathcal{T}
$$

where $\mathcal{T}$ is infinite.

Take a finite ordered tuple $\bar{y}$ of pairwise distinct elements of $\mathcal{T}$ of length $c-2$. We claim that the following elements $\gamma_{1}, \gamma_{2}$ of $\operatorname{IA}_{c-1}(N)$ where

$$
\begin{aligned}
& \begin{array}{rll}
\gamma_{1}: & x_{1} & \mapsto x_{1}\left[x_{1}, \bar{y}, x_{1}\right] \\
& x_{2} & \mapsto x_{2}\left[x_{2}, x_{1}, \bar{y}\right]^{-1}
\end{array} \\
& x_{3} \quad \mapsto x_{3} \\
& \vdots \\
& \begin{array}{ll}
x_{3 k+1} & \mapsto x_{3 k+1}\left[x_{3 k+1}, \bar{y}, x_{1}\right] \\
x_{3 k+2} & \mapsto x_{3 k+2}\left[x_{3 k+2}, x_{1}, \bar{y}\right]^{-1}
\end{array} \\
& x_{3 k+3} \mapsto x_{3 k+3} \\
& t \quad \mapsto t
\end{aligned}
$$

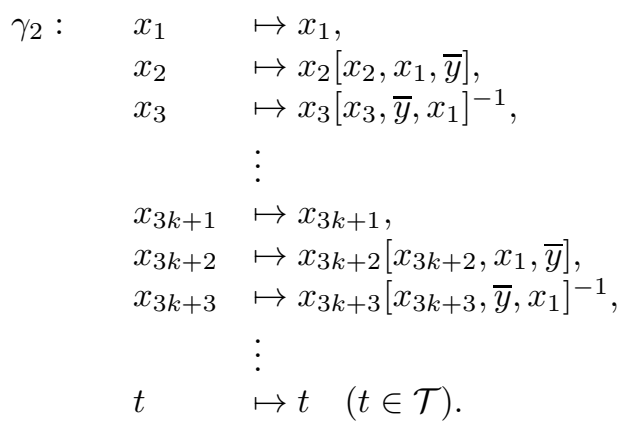

are commutators in the group IA $(N)$. We base our argument on the proof of Lemma 6 of [4. Let $\alpha, \beta \in \operatorname{IA}(N)$ be defined as follows:

$$
\begin{array}{rllll}
\alpha: \quad x_{3 k+1} & \mapsto x_{3 k+1}\left[x_{3 k+2}, x_{1}\right], & \beta: & x_{3 k+1} & \mapsto x_{3 k+1} \\
x_{3 k+2} & \mapsto x_{3 k+2}, & x_{3 k+2} & \mapsto x_{3 k+2}\left[x_{3 k+1}, \bar{y}\right], \\
x_{3 k+3} & \mapsto x_{3 k+3}, & x_{3 k+3} & \mapsto x_{3 k+3}, \\
t & \mapsto t, & t & \mapsto t, \quad(t \in \mathcal{T})
\end{array}
$$

where $k$ is an arbitrary natural number. Using the elegant idea of [4, we first calculate the images of the basis elements under $\alpha \beta$ and then under $\beta \alpha$. Then we introduce elements $\xi_{1}, \xi_{2}$ of $\mathrm{IA}_{c-1}(N)$ such that $\xi_{1}$ takes $x_{3 k+1}$ to $\gamma_{1}\left(x_{3 k+1}\right)(k \in \mathbf{N})$ and fixes all other elements of $\mathcal{B}$ and $\xi_{2}$ which takes $x_{3 k+2}$ to $\gamma_{1}{ }^{-1}\left(x_{3 k+2}\right)(k \in \mathbf{N})$ and again fixes all other elements of $\mathcal{B}$. Next, one sees that the products $\alpha \beta$ and $\beta \alpha$ can be "balanced" by placing $\xi_{1}, \xi_{2}$ into corresponding "pans":

$$
\xi_{1} \alpha \beta=\xi_{2} \beta \alpha .
$$

As $\gamma_{1}=\xi_{1} \xi_{2}{ }^{-1}$, we have the desired. A similar argument proves that $\gamma_{2}$ is also a commutator in the group $\operatorname{IA}(N)$. 
The product $\varepsilon=\gamma_{1} \gamma_{2}$ is

$$
\begin{aligned}
& \varepsilon: \quad x_{1} \quad \mapsto x_{1}\left[x_{1}, \bar{y}, x_{1}\right], \\
& x_{2} \quad \mapsto x_{2}, \\
& x_{3} \mapsto x_{3}\left[x_{3}, \bar{y}, x_{1}\right]^{-1}, \\
& \vdots \\
& x_{3 k+1} \mapsto x_{3 k+1}\left[x_{3 k+1}, \bar{y}, x_{1}\right], \\
& x_{3 k+2} \mapsto x_{3 k+2}, \\
& x_{3 k+3} \mapsto x_{3 k+3}\left[x_{3 k+3}, \bar{y}, x_{1}\right]^{-1} \\
& \vdots \\
& t \quad \mapsto t \quad(t \in \mathcal{T}) .
\end{aligned}
$$

Now the set consisting of $x_{i}$ with $i \neq 1$ going to $x_{i}\left[x_{i}, \bar{y}, x_{1}\right]$ under $\varepsilon$ (say, the set of "pluses"), the set consisting of $x_{i}$ going to $x_{i}\left[x_{i}, \bar{y}, x_{1}\right]^{-1}$ under $\varepsilon$ (the set of "minuses") and the set consisting of $x_{i}$ going themselves are all moieties of $\left\{x_{k}: k \in \mathbf{N}, k \geqslant 1\right\}$. Then there is a permutational automorphism $\pi$ fixing $x_{1}$ and all elements of $\mathcal{T}$ such that

$$
\begin{aligned}
\varepsilon \varepsilon^{\pi}: \quad x_{1} & \mapsto x_{1}\left[x_{1}, \bar{y}, x_{1}\right]^{2}, \\
b & \mapsto b \quad\left(b \in \mathcal{B} \backslash\left\{x_{1}\right\}\right)
\end{aligned}
$$

( $\pi$ must just interchange the set of "pluses" and the set of "minuses"). Let $\delta_{c}$ denote the automorphism

$$
\begin{aligned}
& \delta_{c}: \quad x_{1} \mapsto x_{1}\left[x_{1}, \bar{y}, x_{1}\right], \\
& b \quad \mapsto b \quad\left(b \in \mathcal{B} \backslash\left\{x_{1}\right\}\right)
\end{aligned}
$$

Fix till the end of the proof an automorphism $\Delta$ of the group $\operatorname{Aut}(N)$ stabilizing all conjugations. Due to the remark we have made in the beginning of the proof, $\Delta$ stabilizes the automorphism $\varepsilon \varepsilon^{\pi}=\delta_{c}^{2}$, an element of the commutator subgroup of the group $\operatorname{IA}(N)$. On the other hand, $\Delta\left(\delta_{c}\right)=\delta_{c} \eta$ for some $\eta \in \operatorname{IA}_{c-1}(N)$. Hence

$$
\delta_{c}^{2}=\Delta\left(\delta_{c}^{2}\right)=\delta_{c}^{2} \eta^{2},
$$

whence $\eta=\operatorname{id}_{N}$. Thus $\delta_{c}$ is fixed by $\Delta$.

Recall that given a relatively free group $F$ of infinite rank and a basis $\mathcal{X}$ of $F$, an automorphism $\sigma \in \operatorname{Aut}(F)$ is called finitary relative to $\mathcal{X}$ if $\sigma$ fixes pointwise a cofinite subset of $\mathcal{X}$.

It then follows from Theorem 2.6 that all finitary automorphisms in the group $K=\mathrm{IA}_{c-1}(N)$ (with respect to the basis $\mathcal{B}$ ) are fixed by $\Delta$.

Take an $\eta \in \operatorname{IA}_{c-1}(N)$. We apply Proposition 2.2. Observe that $\eta$ is fully determined by the family of finitary automorphisms $\left\{\eta_{b}: b \in \mathcal{B}\right\}$ such that $\eta_{b} d=d$ for all $d \in \mathcal{B} \backslash\{b\}$ satisfying the conditions

$$
\eta \in \eta_{b} K_{(b)} \quad(b \in \mathcal{B}) .
$$

Now by Proposition 2.2 $\Delta\left(K_{(b)}\right)=K_{(b)}$, since $\Delta$ fixes conjugation $\tau_{b}$, and $\Delta\left(\eta_{b}\right)=$ $\eta_{b}(b \in \mathcal{B})$. Then $\eta$ is stabilized by $\Delta$.

Write $I$ for $\operatorname{IA}(N)$ and $I_{k}$ for $\operatorname{IA}_{k}(N)$. We use downward induction on $k$. Suppose that all elements of the subgroup $I_{k}$ are fixed by $\Delta$ where $k \geqslant 3$. We consider an element

$$
\begin{aligned}
& \delta_{k}: \quad x_{1} \mapsto x_{1}\left[x_{1}, \bar{y}, x_{1}\right], \\
& b \mapsto b \quad\left(b \in \mathcal{B} \backslash\left\{x_{1}\right\}\right)
\end{aligned}
$$


of $I_{k-1}$ where $\bar{y}$ is a tuple of pairwise distinct elements of $\mathcal{T}$ of length $k-2$. Consider the free nilpotent group $\widetilde{N}=N / N_{k+1}$; let denote $\sim$ the homomorphism $\operatorname{Aut}(N) \rightarrow \operatorname{Aut}(\widetilde{N})$, determined by the natural homomorphism $N \rightarrow \widetilde{N}$. As the image of $\delta_{k}^{2}$ under $\sim$ is in the commutator subgroup of the group $\operatorname{IA}(\widetilde{N})$,

$$
\delta_{k}^{2}=\varepsilon \beta
$$

where $\varepsilon$ is in $I^{\prime}$ and $\beta \in I_{k}$. By the induction hypothesis, the automorphism in the right-hand side is fixed by $\Delta$, and then $\delta_{k}$ is also fixed by $\Delta$.

By applying Theorem 2.6 once again, we see that all finitary automorphisms of $N$ lying in the group $I_{k-1}$ are contained in the group

$$
\mathrm{NC}\left(I^{\prime} \cap I_{k-1}, \delta_{k}\right) \cdot I_{k}
$$

and hence all are stabilized by $\Delta$. We then complete the proof as in the case of the subgroup $I_{c-1}$.

\section{Automorphisms of $N$ ThAT FiX A VERY FEW PRIMitive ELEMENTS}

In this section we shall obtain a group-theoretic characterization of the conjugacy class of the permutational automorphism $\pi^{*}$ we have used extensively in the previous section. Recall that $\pi^{*} \in \operatorname{Aut}(N)$ was defined as a permutational automorphism with respect to some basis $\mathcal{X}$ such that there was exactly one element $x \in \mathcal{X}$ that was fixed by $\pi^{*}$, whereas there were infinitely many infinite orbits (cycles) of $\pi^{*}$ on $\mathcal{Y}=\mathcal{X} \backslash\{x\}$. It is easy to see that $x, x^{-1}$ are the only primitive elements fixed by $\pi^{*}$ and conjugations $\tau_{x}, \tau_{x^{-1}}$ are the only conjugations by primitive elements fixed by $\pi^{*}$ under the conjugation action (Claim 3.3). Hence having conjugation by a primitive element fixed by $\pi^{*}$ we can choose in a unique way one of the primitive elements it is determined by. In the next section we shall use this fact to "build" over $\pi^{*}$ the "edifice" of the stabilizer $\Gamma_{(x)}$ of $x$ in the group $\Gamma=\operatorname{Aut}(N)$.

Lemma 3.1. An automorphism $\pi \in \operatorname{Aut}(N)$ is a conjugate of $\pi^{*}$ if and only if

(a) there is a basis set $X$ of conjugations such that some $\tau^{\prime} \in X$ is fixed by $\pi$ and $\pi$ acts on $X \backslash\left\{\tau^{\prime}\right\}$ as a permutation with empty fixed-point set and with infinitely many infinite orbits;

(b) there is a linear order $<$ on $X$ invariant under $\pi$ such that for every $n \geqslant 2$ there is a $\rho_{n} \in \operatorname{Sym}(X)$ which also preserves $<$ and $\pi=\rho_{n}^{n}$.

Proof. $(\Leftarrow)$. We prove the sufficiency part first, partly in order to clarify the role of the condition (b) - for there is no difficulty in the constructing automorphisms $\rho_{n}$ for any conjugate of $\pi^{*}$.

Let us fix some basis $\mathcal{B}$ of $N$. We start with the following observation: suppose there is a linear order $\triangleleft$ on $\mathcal{B}$ which is invariant under some $\sigma \in \operatorname{Sym}(\mathcal{B})$. We then claim that there is a basis $\mathcal{U}_{\sigma}$ of the group $N_{c}$, also invariant under $\sigma$. Such a basis, as we shall see right away, can be constructed with the use of the well-known collecting process.

Let $F(A)$ be a free group with a basis $A$ which is assumed to be linearly ordered. Recall that the collecting process produces the sets $\mathcal{K}_{n}(A)$ of basic commutators of weight $n$ where $n \geqslant 1$. The sets $\mathcal{K}_{n}(A)$ are constructed by induction as follows: $\mathcal{K}_{1}(A)=A$, and to construct the set $\mathcal{K}_{n}(A)$ of basic commutators of weight $n$, we assume that the set $\bigcup_{m<n} K_{m}(A)$ is linearly ordered in a way that the restriction of the order on $A$ is the given one, $u<v$ for every $u \in \mathcal{K}_{m}(A)$ and every $v \in \mathcal{K}_{l}(A)$ 
with $m<l$, and the restriction of the order on the set $\mathcal{K}_{m}(A)$ where $m<n$ could be any. The set $\mathcal{K}_{n}(A)$ is then formed from all commutators $[u, v]$ of weight $n$ such that

$u \in \mathcal{K}_{m}(A), v \in \mathcal{K}_{l}(A)$ with $m+l=n$ and $u>v$, and, moreover,

if $u=\left[u_{1}, u_{2}\right]$, then $u_{2} \leqslant v$.

It can be proven that the image of the set $\mathcal{K}_{m}(A)$ under the natural homomorphism $F(A) \rightarrow L=F(A) / \gamma_{m+1}(F(A))$, from $F(A)$ onto the free nilpotent group $L$ of class $m$, is a basis of the free abelian group $\gamma_{m}(L)$ (see [2, Section 4.3], [16, Section 3.1]). It is then rather clear that the collecting process could be applied to any basis of a free nilpotent group $L$ of class $c$, and the $c$-th step will produce a basis of the $c$-th term $L_{c}$ of the lower central series of $L$.

Let us apply the collecting process to $\mathcal{B}$ to construct a required basis of $N_{c}$ invariant under $\sigma$. The set $\mathcal{K}_{2}(\mathcal{B})$ consists of the commutators $\left[b_{i}, b_{j}\right]$ where $b_{i}, b_{j} \in \mathcal{B}$ such that $b_{i} \triangleright b_{j}$. As the order $\triangleleft$ is invariant under $\sigma, \sigma \mathcal{K}_{2}(\mathcal{B})=\mathcal{K}_{2}(\mathcal{B})$, and, moreover, the lexicographic-like order on $\mathcal{K}_{2}(\mathcal{B})$ (after identifying commutators $[a, b]$ with 2-letter words $a b$ ) is also invariant under $\sigma$.

We then proceed by induction on $m$, constructing the sets $\mathcal{K}_{m}(\mathcal{B})$, where $m \leqslant c$, all equipped with linear orders, namely, with the lexicographical-like orders determined by the orders on the sets $\mathcal{K}_{l}(\mathcal{B})$ with $l<m$. As above, we introduce the lexicographical-like order on $\mathcal{K}_{m}(\mathcal{B})$ where $m \geqslant 3$ by identifying the basic commutators $[u, v]=\left[\left[u_{1}, u_{2}\right], v\right]$ with 3-letter words $u_{1} u_{2} v$. The invariance of these orders under $\sigma$ will guarantee the invariance of the sets $\mathcal{K}_{m}(\mathcal{B})$ under $\sigma$ (for every commutator $[u, v] \in \mathcal{K}_{m}(\mathcal{B})$, the elements $\sigma u, \sigma v$ satisfy the conditions (12), and hence $[\sigma u, \sigma v]$ is in $\left.\mathcal{K}_{m}(\mathcal{B})\right)$.

Next, we deal with a relevant problem on automorphisms of free abelian groups.

Consider a free abelian group $G$ with a basis

$$
\{f\} \cup\left\{e_{k}: k \in \mathbf{Z}\right\} .
$$

Let $H$ denote the subgroup generated by the elements $e_{k}$. Consider an automorphism $\rho$ of $G$ with $\rho\left(e_{k}\right)=e_{k+1}$ for all $k \in \mathbf{Z}$ such that $\rho$ fixes $f$ modulo $H$.

Let $H_{0}$ denote the subgroup of $H$ with the basis

$$
\left\{e_{k}-\rho\left(e_{k}\right): k \in \mathbf{Z}\right\}=\left\{e_{k}-e_{k+1}: k \in \mathbf{Z}\right\} .
$$

Clearly, $H=\left\langle e_{0}\right\rangle \oplus H_{0}$. Suppose that $\rho(f) \not \equiv f\left(\bmod H_{0}\right)$. As, however, $\rho(f) \equiv$ $f(\bmod H)$, then

$$
\rho(f)-f=\alpha e_{0}+h_{0}
$$

where $\alpha$ is a nonzero integer number and $h_{0} \in H_{0}$. We may assume, without loss of generality, that the index of the first nonzero coefficient in the representation of $h_{0}$ as a linear combination of vectors in $(13)$ is $\geqslant 0$. Indeed, let

$$
\rho(f)-f=\alpha e_{0}+\gamma_{m}\left(e_{m}-e_{m+1}\right)+\ldots
$$

where $\gamma_{m}$ is the first nonzero coefficient. We have the desired if $m \geqslant 0$. If, for instance, $m=-1$, then

$\alpha e_{0}+\gamma_{-1}\left(e_{-1}-e_{0}\right)+\ldots=\alpha\left(e_{-1}+e_{0}-e_{-1}\right)+\gamma_{-1}\left(e_{-1}-e_{0}\right)+\ldots=\alpha e_{-1}+\left(\gamma_{-1}-\alpha\right)\left(e_{-1}-e_{0}\right)+\ldots$

If $m<-1$, a trick like that has to be repeated several times.

So let

$$
\rho(f)=f+\alpha e_{0}+\beta_{0}\left(e_{0}-e_{1}\right)+\ldots+\beta_{r}\left(e_{r}-e_{r+1}\right)
$$

where $\beta_{k}$ are integers and, as we assumed before, $\alpha \neq 0$. 
Claim 3.2. Let $n \geqslant 1$ and

$$
\rho^{n}(f)=f+g_{n}
$$

where $g_{n} \in H$. Then $g_{n}$ has at least $n$ nonzero coordinates with regard to the basis $\left\{e_{k}: k \in \mathbf{Z}\right\}$ of $H$.

Proof. Rewriting (14) as

$$
\rho\left(f+\beta_{0} e_{0}+\ldots+\beta_{r} e_{r}\right)=f+\beta_{0} e_{0}+\ldots+\beta_{r} e_{r}+\alpha e_{0}
$$

one easily deduces that

$$
\rho^{n}(f)=f+\alpha e_{0}+\ldots+\alpha e_{n-1}+\beta_{0}\left(e_{0}-e_{n}\right)+\ldots+\beta_{r}\left(e_{r}-e_{r+n}\right),
$$

whence

$$
g_{n}=\alpha e_{0}+\ldots+\alpha e_{n-1}+\beta_{0}\left(e_{0}-e_{n}\right)+\ldots+\beta_{r}\left(e_{r}-e_{r+n}\right) .
$$

Let first $r \leqslant n-1$. Then

$g_{n}=\underbrace{\left(\alpha+\beta_{0}\right) e_{0}+\ldots+\left(\alpha+\beta_{r}\right) e_{r}}_{a}+\alpha e_{r+1}+\ldots+\alpha e_{n-1}-\underbrace{\left(\beta_{0} e_{n}+\ldots+\beta_{r} e_{r+n}\right)}_{b}$.

Suppose that exactly $k$ (out of $r+1$ ) coordinates of the vector $a$ are zero. This means that the vector $b$ has at least $k$ nonzero coordinates. Thus the number of nonzero coordinates of $g_{n}$ is at least

$$
(r+1-k)+(n-1-r)+k=n .
$$

Now let $r \geqslant n$. We have that

$g_{n}=\left(\alpha+\beta_{0}\right) e_{0}+\ldots+\left(\alpha+\beta_{n-1}\right) e_{n-1}+\sum_{k=n}^{r}\left(\beta_{k}-\beta_{k-n}\right) e_{k}-\beta_{r-n+1} e_{r+1}-\ldots-\beta_{r} e_{r+n}$.

Observe that the numbers

$$
r-n+1, \ldots, r
$$

form a complete set of representatives modulo $n$. Let $m$ be an element of this set. Suppose that $m=q n+s$ where $0 \leqslant s<n$ and that

$$
\beta_{m}=0, \beta_{m}-\beta_{m-n}=0, \beta_{m-n}-\beta_{m-2 n}=0, \ldots, \beta_{m-(q-1) n}-\beta_{s}=0 .
$$

It follows that $\beta_{s}=0$, and hence the $s$-th coordinate, that is, $\left(\alpha+\beta_{s}\right)$ is nonzero, or, to put it more generally, there is an $l$ with $l \equiv m(\bmod n)$ such that the $l$-th coordinate is nonzero. Clearly, the latter also holds, if the condition (15) is not true. Thus $g_{n}$ has at least $n$ nonzero coordinates, as claimed.

Let $\pi \in \operatorname{Aut}(N)$ satisfy the conditions (a) and (b). Let $\mathcal{X}$ be a basis of $N$ determining $X$; we assume that $x$ is an element of $\mathcal{X}$ with $\tau^{\prime}=\tau_{x}$.

The order $<$ mentioned in (b) determines the linear order, say $\triangleleft$ of $\mathcal{X}$ :

$$
y \triangleleft z \Longleftrightarrow \tau_{y}<\tau_{z} \quad(y, z \in \mathcal{X}) .
$$

Now the order $\triangleleft$ on $\mathcal{X}$ is invariant under $\pi$ modulo $N_{c}$. According to our considerations above, there is a basis $\mathcal{U}_{\pi}$ of the group $N_{c}$, consisting of basic commutators over $\mathcal{X}$, which is also invariant under $\pi$ (strictly speaking, the arguments above guarantee a basis in question for some $\widetilde{\pi}$ congruent to $\pi$ modulo the subgroup $\mathrm{IA}_{c-1}(N)$; but then the actions of $\pi$ and $\tilde{\pi}$ on $N_{c}$ are identical). Clearly, $\pi$ acts on $\mathcal{U}_{\pi}$ as a permutation with empty fixed-point set and infinitely many infinite orbits. 
As $\pi \tau_{x} \pi^{-1}=\tau_{x}$, we have that $\pi x=x t$ where $t \in N_{c}$. We are going to show that there is a $v \in N_{c}$ with $t=v \pi\left(v^{-1}\right)$. It will follow that $\pi(x v)=x v$, that is, that $\pi$ fixes a primitive element as $\pi^{*}$ does, completing the proof.

Let $n \geqslant 2$ be a natural number. As $\rho_{n}$, a root of $\pi$ of degree $n$, preserves the order on $X$ that $\pi$ does, the basis $\mathcal{U}_{\pi}$ must also be invariant under $\rho_{n}$, and the action of $\rho_{n}$ on this basis must also be similar to that one of $\pi$ : with infinitely many infinite orbits and with empty fixed-point set.

Let

$$
\mathcal{U}_{\pi}=\bigcup_{i \in I} O_{i}
$$

be the partition of $\mathcal{U}_{\pi}$ into $\rho_{n}$-orbits. We are in a position to apply Claim 3.2 to the free abelian group $\left\langle x, N_{c}\right\rangle$ with a basis $\{x\} \cup \mathcal{U}_{\pi}$ and to the restrictions on this group of automorphisms under consideration. Assume that

$$
\rho_{n} x=x \prod_{i \in I} u_{i}^{\alpha_{i}} v_{i} \rho_{n}\left(v_{i}^{-1}\right)
$$

where, of course, only finitely many terms in the latter product are $\neq 1$ and for all $i, u_{i} \in O_{i}, \alpha_{i} \in \mathbf{Z}$, and $v_{i}$ is in the subgroup generated by $O_{i}$.

By Claim 3.2, if at least one of the exponents $\alpha_{i}$ is nonzero, then the length of $t$ with regard to the basis $\mathcal{U}_{\pi}$ is at least $n$. It then follows that there is an $n$ for which $\alpha_{i}$ in (16) are all zero, and hence $\rho_{n}(x)=x v \rho_{n}\left(v^{-1}\right)$ for a suitable $v \in N_{c}$. But this means that $\pi(x v)=\rho_{n}^{n}(x v)=x v$.

$(\Rightarrow)$. Consider the automorphism

$$
f(q)=q+1 \quad(q \in \mathbf{Q})
$$

of $(\mathbf{Q} ;<)$, the set $\mathbf{Q}$ of rational numbers equipped with the natural order $<$. Clearly, $f$ admits extraction of roots of any natural degree $n \geqslant 1$ in the $\operatorname{group} \operatorname{Aut}(\mathbf{Q} ;<)$ : an automorphism $g_{n} \in \operatorname{Aut}(\mathbf{Q} ;<)$ where

$$
g_{n}(q)=q+\frac{1}{n} \quad(q \in \mathbf{Q})
$$

is such that $g_{n}^{n}=f$.

Note that $f$ acts on $\mathbf{Q}$ without fixed points and has infinitely many infinite orbits.

Take a basis $\mathcal{X}=\{x\} \cup \bigcup_{i \in I} \mathcal{Y}_{i}$ of $N$ where $I$ is a linearly ordered set of power $|N|$ and the elements of any set $\mathcal{Y}_{i}$ having countably infinite cardinality are indexed by rational numbers:

$$
\mathcal{Y}_{i}=\left\{y_{i, q}: q \in \mathbf{Q}\right\} \text {. }
$$

We then introduce a linear order $\triangleleft$ on $\mathcal{X}$ by setting that

$$
y_{i, q} \triangleleft x \quad(i \in I, q \in \mathbf{Q})
$$

and that

$$
y_{i, q} \triangleleft y_{i^{\prime}, q^{\prime}} \Longleftrightarrow(i, q)<_{\text {lex }}\left(i^{\prime}, q^{\prime}\right)
$$

for all $i, i^{\prime} \in I$ and $q, q^{\prime} \in \mathbf{Q}$, where $<_{\text {lex }}$ is the lexicographic order on $I \times \mathbf{Q}$.

We define $\pi \in \operatorname{Sym}(\mathcal{X})$ via

$$
\begin{aligned}
& \pi x=x, \\
& \pi y_{i, q}=y_{i, f(q)}, \quad(i \in I, q \in \mathbf{Q}) .
\end{aligned}
$$

As $f \in \operatorname{Aut}(\mathbf{Q} ;<), \pi$ the preserves $\triangleleft$. Clearly, $\pi$ is a conjugate of $\pi^{*}$. 
Let $n \geqslant 1$ be a natural number. Now the automorphism $\rho_{n}$ of $N$ such that

$$
\begin{aligned}
& \rho_{n} x=x, \\
& \rho_{n} y_{i, q}=y_{i, g_{n}(q)}, \quad(i \in I, q \in \mathbf{Q}),
\end{aligned}
$$

is a root of $\pi$ of degree $n$ preserving $\triangleleft$. Finally, both $\pi$ and $\rho_{n}$ preserve the order $<$ on $X=\left\{\tau_{z}: z \in \mathcal{X}\right\}$ where

$$
\tau_{z}<\tau_{z^{\prime}} \Longleftrightarrow z \triangleleft z^{\prime}
$$

for all $z, z^{\prime} \in \mathcal{X}$.

We record the following simple observation about $\pi^{*}$ for future reference.

Claim 3.3. Let $\mathcal{X}$ be a basis of $N$ such that $\pi^{*} \in \operatorname{Sym}(\mathcal{X})$ and there is exactly one element $x \in \mathcal{X}$ fixed by $\pi^{*}$, while the action of $\pi^{*}$ on $\mathcal{X} \backslash\{x\}$ is one with infinitely many infinite orbits. Then $x$ and $x^{-1}$ are the only primitive elements fixed by $\pi^{*}$ (fixed by $\pi^{*}$ up to congruence modulo any subgroup $N_{k}$ where $2 \leqslant k \leqslant c$ ).

Proof. It is convenient to prove the statement by induction on $c$. When $c=1, N$ is a free abelian group and the result is obvious. Suppose that $\pi^{*}(a)=a$ where $a$ is a primitive element of $N$. Consider the natural homomorphism ${ }^{\wedge}: N \rightarrow N / N_{c}$. This homomorphism determines the homomorphism $\operatorname{Aut}(N) \rightarrow \operatorname{Aut}\left(N / N_{c}\right)$ which

we denote by the same symbol $\widehat{~}$. Then $\widehat{\pi^{*}}(\widehat{a})=\widehat{a}$, whence $\widehat{a}=\widehat{x}^{ \pm 1}$. Therefore $a=x^{ \pm 1} t$, where $t=t(x, \bar{y}) \in N_{c}$ and $\bar{y}$ is a tuple of element of $\mathcal{X} \backslash\{x\}$. It follows that

$$
t\left(x,\left(\pi^{*}\right)^{k} \bar{y}\right)=t(x, \bar{y})
$$

for every $k \in \mathbf{Z}$ and then $t(x, \bar{y})=1$.

\section{Reconstruction of PRimitive elements}

The aim of this section is to show definability of stabilizers $\Gamma_{(x)}$ of primitive elements of $N$ where $\Gamma=\operatorname{Aut}(N)$.

Theorem 4.1. (i) Let $z$ be a primitive element of $N$ which is fixed by $\pi^{*}$. Then the group $\Gamma_{(z)}$ of all automorphisms of $N$ stabilizing $z$ is definable with the parameter $\pi^{*}$

(ii) the family of stabilizers of primitive elements of $N$ is invariant under the action of the group $\operatorname{Aut}(\operatorname{Aut}(N))$.

Proof. (i). We first note that by Claim $3.3 \pi^{*}$ fixes exactly two primitive elements that are inverses of one another. So the element $z$ in the condition of (i) can equivalently be replaced with $z^{-1}$, because $\Gamma_{(z)}=\Gamma_{\left(z^{-1}\right)}$.

Let $\tau^{*}$ be conjugation by a primitive elements stabilized by $\pi^{*}$ under the conjugation action. Again, it follows from Claim 3.3 (for the case when $k=c$ ) that there are exactly two such conjugations, inverses of one another. Let $x$ be the (only) primitive element of $N$ such that $\pi^{*}(x)=x$ and $\tau^{*}=\tau_{x}$. Recall that we write $Z^{+}\left(\pi^{*}\right)$ for the subgroup of the centralizer $Z\left(\pi^{*}\right)$ of $\pi^{*}$ consisting of automorphisms of $N$ that fix $x$. Then the subgroup $Z^{+}\left(\pi^{*}\right)$ is definable with the parameter $\pi^{*}$, for $\sigma \in Z^{+}\left(\pi^{*}\right)$ if and only if $\sigma$ preserves $\tau^{*}$ under the conjugation action and commutes with $\pi^{*}$.

Let us fix a basis $X$ of $\operatorname{Inn}(N)$ containing conjugation $\tau^{*}$ and a basis $\mathcal{X}$ of $N$, containing $x$, which determines $X$. Write $\mathcal{Y}$ for the set $\mathcal{X} \backslash\{x\}$. 
We are going to prove that the stabilizer $\Pi_{(x)}$ of $x$ in the group $\Pi=\operatorname{Sym}(\mathcal{X})$ is contained in a definable with the parameter $\pi^{*}$ subgroup of $\operatorname{Aut}(N)$. We shall then demonstrate that the subgroup $\Pi_{(x)}$ and the subgroup $Z^{+}\left(\pi^{*}\right)$ generate the required subgroup $\Gamma_{(x)}$ :

$$
\Gamma_{(x)}=\left\langle\Pi_{(x)}, Z^{+}\left(\pi^{*}\right)\right\rangle .
$$

All actions mentioned in the following lemma are actions on $X$ by conjugation.

Lemma 4.2. (a) Let $\psi \in \operatorname{Sym}(X)$ be an involution which fix $\tau^{*}=\tau_{x}$ and which acts on $X$ as a transposition interchanging distinct conjugations $\tau_{y}=\tau_{y}(\psi)$ and $\tau_{z}=\tau_{z}(\psi)$ of $X$ where $y, z \in \mathcal{Y}$. Suppose that $\sigma$ is a power of $\pi^{*}$ such that $\tau_{y}$ and $\tau_{z}$ do not lie in the same orbit of $\sigma$ and for every natural number $n \geqslant 1$ there is a $\rho_{n} \in \operatorname{Sym}(X)$ having the following properties:

- $\rho_{n}$ commutes with the $n$-th power $\sigma^{n}$ of $\sigma$;

- the orbits $O_{n}, O_{n}^{\prime} \subseteq X$ of $\sigma^{n}$ containing $\tau_{y}$ and $\tau_{z}$, respectively, are both fixed pointwise by $\rho_{n}$;

- the actions of $\sigma^{n}$ and $\rho_{n}$ on $X \backslash\left(O_{n} \cup O_{n}^{\prime}\right)$ are the same;

- $\psi$ commutes with $\rho_{n}$.

Then $\psi x=x t$ where $t$ is an element of $N_{c} \cap\langle x, y, z\rangle$.

(b) Suppose that for an involution $\psi$ satisfying the conditions of (a) there is an involution $\rho \in \operatorname{Sym}(X)$, commuting with a power of $\pi^{*}$, which takes $\tau_{y}(\psi)$ to $\tau_{z}(\psi)$ and commutes with $\psi$. Then $\psi x=x$.

(c) Let $\Psi \subseteq \operatorname{Sym}(X)$ be a family of involutions satisfying the conditions of (b) such that for every distinct $y, z \in \mathcal{Y}$ there is exactly one element of $\Psi$ taking $\tau_{y}$ to $\tau_{z}$. Suppose that an involution $\varphi \in \operatorname{Sym}(X)$ preserves $\tau^{*}$ and normalizes $\Psi$ : $\varphi \Psi \varphi=\Psi$. Then $\varphi x=x$.

Proof. (a) Assume that $\psi x=x t$ where $t \in N_{c}$ and that $t$ is written as a reduced word in letters of $\mathcal{X}$.

We work with the case when $n=1$. Note that being an element of $\operatorname{Sym}(X), \rho_{1}$ cannot invert $\tau^{*}$. Then as $\rho_{1}$ commutes with $\sigma$, we have that $\rho_{1} x=x$. Further, $\rho_{1} \psi \rho_{1}^{-1}=\psi$ implies that $x \rho_{1}(t)=x t$, or $\rho_{1}(t)=t$. Now $\rho_{1}$ acts a permutation having no fixed points and having infinitely many infinite orbits on $X \backslash\left(\left\{\tau_{x}\right\} \cup\right.$ $O_{1} \cup O_{1}^{\prime}$ ). Let subsets $\mathcal{O}_{1}$ and $\mathcal{O}_{1}^{\prime}$ of $\mathcal{X}$ induce the sets $O_{1}$ and $O_{1}^{\prime}$. It then follows that the reduced word $t$ has no occurrences of letters in $\mathcal{X} \backslash\left(\{x\} \cup \mathcal{O}_{1} \cup \mathcal{O}_{1}^{\prime}\right)$, or $t \in N_{c} \cap\left\langle\{x\} \cup \mathcal{O}_{1} \cup \mathcal{O}_{1}^{\prime}\right\rangle$.

Let $\mathcal{O}_{n}, \mathcal{O}_{n}^{\prime}$ be the subsets of $\mathcal{X}$ determining the sets $O_{n}, O_{n}^{\prime}$, respectively. Arguing as above, we see that $t \in N_{c} \cap\left\langle\{x\} \cup \mathcal{O}_{n} \cup \mathcal{O}_{n}^{\prime}\right\rangle$ for all $n \geqslant 1$. Observe that

$$
\bigcap_{n \geqslant 1}\left(\mathcal{O}_{n} \cup \mathcal{O}_{n}^{\prime}\right)=\{y, z\}
$$

Hence $t=t(x, y, z)$.

(b). As $\psi$ is an involution, then $\psi^{2} x=x$ implies that $x t(x, y, z) t(x, z, y)=x$, whence $t(x, z, y)=t(x, y, z)^{-1}$. Let $\rho$ satisfy the conditions of (b). Then

$$
\rho \psi \rho(x)=\psi x \Longleftrightarrow \rho[x t(x, y, z)]=x t(x, y, z) \Longleftrightarrow x t(x, z, y)=x t(x, y, z) .
$$

Then $t(x, y, z)^{2}=1$, and $t=1$, as claimed.

(c) Suppose that $\varphi x=x s$ where $s \in N_{c}$. Clearly, $\varphi$ sends $s$ to the inverse. Choose an arbitrary $\psi \in \Psi$. We then have that $\varphi \psi \varphi=\psi^{\prime}$ for a suitable $\psi^{\prime} \in \Psi$. 
Then $\varphi \psi \varphi x=\psi^{\prime} x=x$. Therefore

$$
x=\varphi \psi \varphi(x)=\varphi \psi(x s)=\varphi(x \psi s)=x \cdot s \cdot \varphi \psi(s),
$$

or

$$
\varphi \psi(s)=s^{-1} \Longleftrightarrow \psi(s)=\varphi\left(s^{-1}\right) \Longleftrightarrow \psi(s)=s .
$$

Thus $\psi(s)=s$ for all $\psi \in \Psi$. This easily implies that $s=1$, for the subgroup of $\operatorname{Sym}(X)$ generated by $\Psi$ acts on $\mathcal{X} \backslash\{x\}$ modulo $N_{c} k$-transitively for every natural number $k \geqslant 1$.

Now we are constructing a promised definable with the parameter $\pi^{*}$ subgroup $S$ containing the group $\Pi_{(x)}$. Statements of Lemma 4.2 suggest the construction of $S$ as the subgroup of $\operatorname{Aut}(N)$ generated by all involutions in $\operatorname{Sym}(X)$ that are in the normalizers of families involutions satisfying the conditions of (c):

$$
S=\left\langle\varphi \in \operatorname{Sym}(X): \varphi^{2}=\mathrm{id}, \varphi \tau^{*} \varphi=\tau^{*}, \exists \Psi \text { with (c) s.t. } \varphi \Psi \varphi=\Psi\right\rangle .
$$

By Lemma $4.2, \sigma x=x$ for all $\sigma \in S$. Let us show that

$$
S \supseteq \Pi_{(x)}=\{\sigma \in \operatorname{Sym}(\mathcal{X}): \sigma x=x\} .
$$

Indeed, consider the family $\Psi^{*}$ of transpositions $\psi_{y, z} \in \operatorname{Sym}(\mathcal{X})$ where distinct $y, z$ run over $\mathcal{Y}$ such that $\psi_{y, z}$ interchanges $y$ with $z$ and fixes all other elements of $\mathcal{X}$.

Let us check that $\Psi^{*}$ satisfies (c). Take any $\psi_{y, z} \in \Psi^{*}$. The check of (a) for $\psi_{y, z}$ is simple: one easily finds a power $\sigma$ of $\pi^{*}$ such $y, z$ lie in the different orbits of $\sigma$. Then the construction of a needed $\rho_{n}$ for every $n \geqslant 1$ is trivial. Let us check (b). Consider as a $\rho$ an element of $\operatorname{Sym}(\mathcal{X})$ which fixes $x$, and sends $y$ to $z$ while interchanging the orbits of $\sigma$ on $\mathcal{X}$ containing $y$ and $z$; all other orbits may, say stay fixed pointwise under the action of $\rho$. Hence the conditions of (b) are also true for $\psi_{y, z}$.

Recall that any infinite symmetric group is generated by involutions (as a bit too powerful tool one can apply, for instance, Baer's theorem on the structure of normal subgroups of the infinite symmetric group $\operatorname{Sym}(\Omega)$ [7, p. 256]; it is an immediate corollary of the said theorem that the normal closure of any element of $\operatorname{Sym}(\Omega)$-in particular, any involution - whose support is of power $|\Omega|$ is the whole of $\operatorname{Sym}(\Omega)$ ).

Now as any involution $\varphi$ of the group $\Pi_{(x)}$ normalizes $\Psi^{*}$, the group $\Pi_{(x)}$ is contained in $S$.

Let us again consider a relevant problem on free abelian groups. Let $G$ be a free abelian group with a basis $\{a\} \cup\left\{b_{i}: i \in I\right\}$. Let $H$ denote the subgroup generated by $\left\{b_{i}: i \in I\right\}$. Then an arbitrary automorphism $\beta$ of $G$ fixing $a$ looks like

$$
\begin{aligned}
& \beta a=a \\
& \beta b_{i}=c_{i}+k_{i} a, \quad(i \in I)
\end{aligned}
$$

where $c_{i}$ is in $H$ and $k_{i} \in \mathbf{Z}(i \in I)$. Clearly, if $\alpha \in \operatorname{Aut}(G)$ is defined via

$$
\begin{aligned}
& \alpha a=a \\
& \alpha b_{i}=b_{i}-k_{i} a, \quad(i \in I),
\end{aligned}
$$

then $\beta \alpha \in \operatorname{Aut}(G)_{(a),\{H\}}$.

This observation implies that

$$
\Gamma_{(x)}=\Gamma_{(x),\{\langle\mathcal{Y}\rangle\}} \cdot A_{x} \cdot \operatorname{IA}(N)_{(x)} .
$$


where $A_{x}$ is the group of all automorphisms of $N$ of the form

$$
\begin{aligned}
& \alpha x=x \\
& \alpha y=y x^{k_{y}}, \quad(y \in \mathcal{Y})
\end{aligned}
$$

where $k_{y} \in \mathbf{Z}$ for all $y \in \mathcal{Y}$.

We then claim that

$$
\Gamma_{(x)}=\left\langle\Pi_{(x)}, Z^{+}\left(\pi^{*}\right)\right\rangle .
$$

This will complete the proof of (i).

Indeed, the group $\operatorname{IA}(N)_{(x)}=\operatorname{IA}_{1}(N)_{(x)}$ is contained in the group $\left\langle\Pi_{(x)}, Z^{+}\left(\pi^{*}\right)\right\rangle$ by Lemma 2.5. Let $\mathcal{Z}$ be any moiety of $\mathcal{Y}$. It is easy to see that $A_{x}$ equals the product the subgroup $\left(A_{x}\right)_{(\mathcal{X} \backslash \mathcal{Z})}$ and the conjugate subgroup of $\left(A_{x}\right)_{(\mathcal{X} \backslash \mathcal{Z})}$ by a suitable element of $\Pi_{(x)}$. But then, by Lemma 2.3, the group $\left\langle\Pi_{(x)}, Z^{+}\left(\pi^{*}\right)\right\rangle$ contains the group $\left(A_{x}\right)_{\left(\mathcal{X} \backslash \mathcal{Z}_{0}\right)}$ for a suitable moiety $\mathcal{Z}_{0}$ of $\mathcal{Y}$.

The same lemma implies that the group $\left\langle\Pi_{(x)}, Z^{+}\left(\pi^{*}\right)\right\rangle$ contains the subgroup $\Gamma_{\left\langle\mathcal{Z}_{0}\right\rangle}$ where $\mathcal{Z}_{0}$ is a moiety of $\mathcal{Y}$. As the author showed in [20, given any partition $\mathcal{X}=\mathcal{X}_{1} \cup \mathcal{X}_{2} \cup \mathcal{T}$ of $\mathcal{X}$ into pairwise disjoint moieties, we have that the group $\Gamma=\operatorname{Aut}(N)$ is generated by the stabilizers

$$
\Gamma_{\left(\mathcal{X}_{1}\right),\left\{\left\langle\mathcal{X}_{2}, \mathcal{T}\right\rangle\right\}} \text { and } \Gamma_{\left(\mathcal{X}_{2}\right),\left\{\left\langle\mathcal{X}_{1}, \mathcal{T}\right\rangle\right\}} .
$$

(Theorem 2.5 in [20]). Clearly, the stabilizers in (19) are conjugate by a suitable permutational automorphism of $N$ with respect to the basis $\mathcal{X}$. Thus $\operatorname{Aut}(N)$ is generated by some $\rho \in \operatorname{Sym}(\mathcal{X})$ and the stabilizer $\Gamma_{\left\langle\mathcal{X}_{2}, \mathcal{T}\right\rangle}=\Gamma_{\left(\mathcal{X}_{1}\right),\left\{\left\langle\mathcal{X}_{2}, T\right\rangle\right\}}$.

By applying these considerations to the situation at hands, we see that the subgroup $\Gamma_{(x),\{\langle\mathcal{Y}\rangle\}} \cong \operatorname{Aut}(\langle\mathcal{Y}\rangle)$ is generated by an element of $\Pi_{(x)}$ and the group $\Gamma_{\left\langle\mathcal{Z}_{0}\right\rangle} \subseteq\left\langle\Pi_{(x)}, Z^{+}\left(\pi^{*}\right)\right\rangle$.

(ii) Let $\Delta \in \operatorname{Aut}(\operatorname{Aut}(N))$ and $\Sigma \subseteq \operatorname{Aut}(N)$ be the stabilizer of a primitive element of $N$. Then $\Delta(\Sigma)$ is also a stabilizer of a primitive element of $N$ by Lemma 3.1 and (i).

\section{Stabilizing EVERYThing}

The family of all pairs $(\tau, \Sigma)$ where $\tau$ and $\Sigma$ satisfy the condition

" $\tau$ is conjugation determined by a primitive element of $N$ and $\Sigma \subseteq \operatorname{Aut}(N)$ is the stabilizer of a primitive element such that every element of $\Sigma$ commutes with $\tau$ "

is a definable object over $\operatorname{Aut}(N)$ by Theorem 1.4. Lemma 3.1 and Theorem 4.1 We shall call pairs with (20) primitive pairs.

Clearly, every primitive pair $(\tau, \Sigma)$ uniquely determines some primitive element $p(\tau, \Sigma)$ of $N$. Indeed, a conjugation $\tau$ determines the coset $x N_{c}$ where $\tau=\tau_{x}$, consisting of primitive elements and we then choose the only element $x s$ where $s \in N_{c}$ which is stabilized by every member of $\Sigma$.

Now the conjugation action

$$
\sigma *(\tau, \Sigma)=\left(\sigma \tau \sigma^{-1}, \sigma \Sigma \sigma^{-1}\right)
$$

of the group $\operatorname{Aut}(N)$ on the set of all primitive pairs is equivalent to the action of the group $\operatorname{Aut}(N)$ on the set of all primitive elements of $N$.

We are going to show that the multiplication of "independent" primitive elements is definable. So let us take two pairs $\left(\tau_{1}, \Sigma_{1}\right)$ and $\left(\tau_{2}, \Sigma_{2}\right)$ with $(20)$ which determine primitive elements $x$ and $y$ of $N$, respectively. To explain that $x$ and $y$ 
are independent, we require that there be a basis set of conjugations into which both conjugations $\tau_{1}, \tau_{2}$ can be included.

Lemma 5.1. The primitive pair $\left(\tau_{1} \tau_{2}, \Sigma_{1,2}\right)$ associated with the product $x y$ of the elements $x=p\left(\tau_{1}, \Sigma_{1}\right)$ and $y=p\left(\tau_{2}, \Sigma_{2}\right)$ is definable with the parameters $\left(\tau_{1}, \Sigma_{1}\right),\left(\tau_{2}, \Sigma_{2}\right)$.

Proof. Let $X$ be any basis set of conjugations that contains both $\tau_{1}$ and $\tau_{2}$. For every $\tau \in X \backslash\left\{\tau_{1}, \tau_{2}\right\}$ we choose a pair $\left(\tau, \Sigma_{\tau}\right)$ with (20) determining an element $z_{\tau}$, thereby defining a basis

$$
\mathcal{X}=\{x, y\} \cup\left\{z_{\tau}: \tau \in X \backslash\left\{\tau_{1}, \tau_{2}\right\}\right\}
$$

of $N$.

Consider a pair $\left(\tau_{1} \tau_{2}, \Sigma\right)$ satisfying $(20)$. It is clear that this pair determines a primitive element $p$ of the form $p=x y t$ where $t \in N_{c}$. Let $w\left(*_{1}, *_{2}\right)$ be a group word in two letters over an alphabet having no common elements with $N$ (or a term of the language of group theory having two variables) such that $t=w(x, y)$ where $w(x, y)$ is the result of replacing letters/variables of $w$ to $x$ and $y: *_{1} \rightarrow x, *_{2} \rightarrow y$.

Observe that given two distinct elements $z_{1}, z_{2}$ of $\mathcal{X}$, the element $r=z_{1} z_{2} w\left(z_{1}, z_{2}\right)$ is definable over $\mathcal{X}$ and $p$, for $r$ is the result of action on $p=x y w(x, y)$ by a permutational automorphism with respect to $\mathcal{X}$ which takes $x$ to $z_{1}$ and $y$ to $z_{2}$. For instance, the element $r=y x w(y, x)$ must be described as follows: first, we are describing the uniquely determined $\pi \in \operatorname{Aut}(N)$ such that

$$
\begin{aligned}
& \pi *\left(\tau_{1}, \Sigma_{1}\right)=\left(\tau_{2}, \Sigma_{2}\right), \\
& \pi *\left(\tau_{2}, \Sigma_{2}\right)=\left(\tau_{1}, \Sigma_{1}\right), \\
& \pi *\left(\tau, \Sigma_{\tau}\right)=\left(\tau, \Sigma_{\tau}\right), \quad\left(\tau \in X \backslash\left\{\tau_{1}, \tau_{2}\right\}\right),
\end{aligned}
$$

and then set the pair $R$ corresponding to $r$ as $R=\pi *\left(\tau_{1} \tau_{2}, \Sigma\right)$. On similar occasions below, we will just describe actions on primitive elements themselves, skipping translations into the language of action on primitive pairs.

Our first condition, we are going to impose on the pair $\left(\tau_{1} \tau_{2}, \Sigma\right)$, will imply that $w(x, y)=w(y, x)$. To achieve that we require the following automorphism $\alpha$ defined via

$$
\begin{aligned}
\alpha: \quad & x \mapsto x \\
& y x w(y, x) \mapsto x y w(x, y), \\
& z x w(z, x) \mapsto x z w(x, z), \quad z \in \mathcal{X} \backslash\{x, y\}
\end{aligned}
$$

be equal to $\tau_{1}=\tau_{x}$. It then follows that

$$
\begin{aligned}
& \alpha(x)=x \\
& \alpha(y)=x y x^{-1} w(x, y) w(y, x)^{-1}, \\
& \alpha(z)=x z x^{-1} w(x, z) w(z, x)^{-1}, \quad z \in \mathcal{X} \backslash\{x, y\}
\end{aligned}
$$

whence

$$
w(x, y)=w(y, x)
$$

as claimed. Clearly, as $x, y$ are members of some basis of $N$, then $w(a, b)=w(b, a)$ for every $a, b \in N$ (apply to the both parts of (21) an endomorphism of $N$ taking $x$ to $a$ and $y$ to $b$.)

Fix a $z$ from $\mathcal{X} \backslash\{x, y\}$ and write $q$ for $x z w(x, z)$. Consider the automorphisms

$$
\begin{aligned}
U_{1}: & y \mapsto y z w(y, z), \\
& a \mapsto a, \quad(a \in \mathcal{X} \backslash\{y\})
\end{aligned}
$$


and

$$
\begin{aligned}
U_{2}: & x \mapsto x y w(x, y), \\
& a \mapsto a, \quad(a \in \mathcal{X} \backslash\{x\}) ;
\end{aligned}
$$

We then require $U_{1}(p)$ and $U_{2}(q)$ be equal:

$$
U_{1}(p)=x y z w(y, z) w(x, y z)=U_{2}(q)=x y z w(x, y) w(x y, z) .
$$

Therefore

$$
w(x, y) w(x y, z)=w(y, z) w(x, y z) .
$$

Fix a natural number $k$ and apply the endomorphism of $N$ taking $y$ to $x^{k}$ and fixing all other elements of $\mathcal{X}$ to the both parts of the last equation. It then follows that

$$
w\left(x^{k+1}, z\right)=w\left(x^{k}, z\right) w\left(x, x^{k} z\right) .
$$

We apply a permutational automorphism with respect to $\mathcal{X}$ interchanging $x$ and $z$ to the both parts of $(22)$ :

$$
w\left(z^{k+1}, x\right)=w\left(z^{k}, x\right) w\left(z, z^{k} x\right) .
$$

Due to "symmetricity" of $w$ we then have that

$$
w\left(x, z^{k+1}\right)=w\left(x, z^{k}\right) w\left(z^{k} x, z\right) .
$$

Multiplying the latter equation with (22) part by part, we arrive at

$$
w\left(x^{k+1}, z\right) w\left(x, z^{k+1}\right)=w\left(x^{k}, z\right) w\left(x, z^{k}\right) \cdot w\left(x^{k} z, x\right) w\left(z^{k} x, z\right) .
$$

Then by (5),

$$
w(x, z)^{(k+1)^{c}}=w(x, z)^{k^{c}} w\left(x z^{k}, z\right) w\left(z x^{k}, x\right),
$$

or

$$
w(x, z)^{(k+1)^{c}-k^{c}}=w\left(x z^{k}, z\right) w\left(z x^{k}, x\right) .
$$

Let $L_{k}=\left\{s^{k}: s \in N_{c}\right\}$ be the subgroup of all $k$-th powers of elements of the (free abelian) group $N_{c}$. As $(k+1)^{c}-k^{c} \equiv 1(\bmod k)$ and as, for instance, $w\left(x z^{k}, z\right) \equiv$ $w(x, z)\left(\bmod L_{k}\right)$, we get that

$$
w(x, z) \equiv 1\left(\bmod L_{k}\right)
$$

for every natural number $k$. Therefore $w(x, z)=1$, which means that the element $x y t$ determined by the pair $\left(\tau_{1} \tau_{2}, \Sigma\right)$ is $x y$, as desired.

REMARK. The definability of $x y$ over $\{x, y\}$ is much more simple when the nilpotency class $c$ of $N$ is even. Indeed, extend $\{x, y\}$ to a basis $\mathcal{X}$ of $N$ and consider the automorphism $\theta \in \operatorname{Aut}(N)$ that inverts all elements of $\mathcal{X}$. Then the element $x y t$ where $t \in N_{c}$ is inverted by the automorphism $\tau_{x} \theta$ if and only if $t=1$. This condition can be easily translated into the language of the action of $\operatorname{Aut}(N)$ on the primitive pairs.

Theorem 5.2. The group $\operatorname{Aut}(N)$ is complete.

Proof. According to Proposition 1.2 the group $\operatorname{Aut}(N)$ is centreless.

Let $\Delta \in \operatorname{Aut}(\operatorname{Aut}(N))$. By Proposition 1.9 there is a $\sigma_{0} \in \operatorname{Aut}(N)$ such that $\Delta_{1}=T_{\sigma_{0}} \circ \Delta$ stabilizes all elements of the group $\operatorname{Inn}(N)$ and, according to the same Proposition,

$$
\Delta_{1}(\sigma) \equiv \sigma\left(\bmod \mathrm{IA}_{c-1}(N)\right) \quad(\sigma \in \operatorname{Aut}(N)) .
$$


Take a basis set $X$ of conjugations, and then for each $\tau \in X$ choose the stabilizer $\Sigma_{\tau}$ of a primitive element, forming a primitive pair $\left(\tau, \Sigma_{\tau}\right)$. Let $P(X)$ denote the set

$$
\left\{\left(\tau, \Sigma_{\tau}\right): \tau \in X\right\}
$$

and let $\mathcal{X}$ be a basis of $N$ determined by the pairs in $P(X)$. Suppose that a pair $\left(\tau, \Sigma_{\tau}\right)$ determines the primitive element $x_{\tau}$.

Take a $\tau \in X$. The image of the pair $\left(\tau, \Sigma_{\tau}\right)$ under $\Delta_{1}$ is a pair $\left(\tau, \Sigma_{\tau}^{*}\right)$ which determines a primitive element $x_{\tau} t_{\tau}$ where $t_{\tau} \in N_{c}$. Indeed, let $\pi$, a conjugate of $\pi^{*}$, belong to $\Sigma_{\tau}$; then $\pi \tau \pi^{-1}=\tau$ by the definition of a primitive pair. By (23),

$$
\Delta_{1}(\pi) \tau \Delta_{1}(\pi)^{-1}=\tau .
$$

On the other hand, $\Delta_{1}(\pi)$ must be a conjugate of $\pi^{*}$ (Lemma 3.1) and hence $\Delta(\pi)$ fixes a uniquely determined element in the $\operatorname{coset} x_{\tau} N_{c}$, the above mentioned element $x_{\tau} t_{\tau}$.

Consider the automorphism $\alpha \in \mathrm{IA}_{c-1}(N)$ which takes $x_{\tau}$ to $x_{\tau} t_{\tau}(\tau \in X)$. Then $\Delta_{2}=T_{\alpha^{-1}} \circ \Delta_{1}$ stabilizes all pairs $\left(\tau, \Sigma_{\tau}\right)$ where $\tau$ runs over $X$.

Lemma 5.3. $\Delta_{2}$ (and hence already $\left.\Delta_{1}\right)$ stabilizes all elements of the group $\operatorname{IA}(N)$.

Proof. Let $x, y$ be two distinct elements of $\mathcal{X}$. Consider the IA-automorphism $K_{12}$ (the standard notation) defined via

$$
\begin{aligned}
& K_{12}(x y)=y x \Longleftrightarrow K_{12}(x)=y x y^{-1}, \\
& K_{12} z=z, \quad(z \in \mathcal{X} \backslash\{x\}) .
\end{aligned}
$$

Then as the pairs associated with the elements $x y$ and $y x$ are definable over $P(X)$ and $P(X)$ is stabilized by $\Delta_{2}, K_{12}$ is stabilized by $\Delta_{2}$.

According to the classical results of Nielsen and Magnus, the normal closure of an analogue of $K_{12}$ in the automorphism group $\operatorname{Aut}\left(F_{n}\right)$, where $F_{n}(n \geqslant 2)$ is a finitely generated free group, is the group $\mathrm{IA}\left(F_{n}\right)$ [15, Section 3.5]. Also, the homomorphism $\operatorname{Aut}\left(F_{n}\right) \rightarrow \operatorname{Aut}\left(F_{n} / \gamma_{3}\left(F_{n}\right)\right)$, determined by the natural homomorphism $F_{n} \rightarrow F_{n} / \gamma_{3}\left(F_{n}\right)$, is surjective [1] (equivalently, one says that the automorphism groups of finitely generated free nilpotent groups of class two are tame). It follows that in the automorphism group of any infinitely generated free nilpotent group of class two all finitary IA-automorphisms are contained in the normal closure of any analogue of $K_{12}$. Therefore finitary IA-automorphisms of $N$ are all contained in the group $\mathrm{NC}\left(K_{12}\right) \cdot \mathrm{IA}_{2}(N)$.

By Proposition 1.9. all elements of $\mathrm{NC}\left(K_{12}\right)$ are stabilized by $\Delta_{2}$ and the group $\mathrm{IA}_{2}(N)$ is stabilized by $\Delta_{2}$ by Proposition 2.7. Thus all finitary IA-automorphisms of $N$ are stabilized by $\Delta_{2}$, and by using the fact that subgroups $\operatorname{IA}(N)_{(p)}$ where $p$ is any primitive element of $N$ are invariant under $\Delta_{2}$ (Proposition 2.2), we can complete the proof as in the proof of Proposition 2.7

Swan (see [6, Section 2]) found rather simply-constructed generating sets of the automorphism groups of infinitely generated free abelian groups. Let $A$ be an infinitely generated free abelian group and let $\mathcal{B}$ be a basis of $A$. An automorphism $\theta$ of $A$ is called $\mathcal{B}$-block-unitriangular if there is a moiety $\mathcal{C}$ of $\mathcal{B}$ such that $\theta$ fixes $\mathcal{C}$ pointwise and

$$
\theta d \equiv d(\bmod \langle\mathcal{C}\rangle) .
$$

for all $d \in \mathcal{B} \backslash \mathcal{C}$. Then the group $\operatorname{Aut}(A)$ is generated by all $\mathcal{B}$-block-unitriangular automorphisms. 
Let $\mathcal{Y}$ be a moiety of $\mathcal{X}$. Consider then an automorphism $\beta$ of $N$ which induces a block-unitriangular automorphism in the abelianization of $N$ :

$$
\begin{aligned}
& \beta z=z \prod_{y \in \mathcal{Y}} y^{m(y, z)} \quad(z \in \mathcal{X} \backslash \mathcal{Y}), \\
& \beta y=y \quad(y \in \mathcal{Y})
\end{aligned}
$$

where in any (formal) product $\prod_{y \in \mathcal{Y}} y^{m(y, z)}$ only finitely many integer exponents $m(y, z)$ are non-zero.

By Swan's theorem, the group Aut $(N)$ is generated by the automorphisms of the form (24) and by the elements of the group $\operatorname{IA}(N)$. It therefore remains to prove that $\Delta_{2}$ stabilizes all automorphisms of the form (24).

We use Lemma 5.1. Any element of the form $z \prod_{y \in \mathcal{Y}} y^{m(y, z)}$ can be obtained from elements of $\mathcal{X}$ as a result of a number of successive applications of the operation "multiplication of independent primitives" like in the following example:

$$
z \rightarrow z \cdot y_{1}^{-1} \rightarrow z y_{1}^{-1} \cdot y_{1}^{-1} \rightarrow z y_{1}^{-2} \cdot y_{2} \rightarrow \ldots \rightarrow z y_{1}^{-2} y_{2}^{2} \cdot y_{2} \rightarrow \ldots
$$

Therefore the image under $\beta \in \operatorname{Aut}(N)$ with (24) of any basis pair $\left(\tau, \Sigma_{\tau}\right)$ that determines an element of $\mathcal{X} \backslash \mathcal{Y}$ is a primitive pair definable with the parameters from the set of basis pairs $P(X)$. As $\Delta_{2}$ fixes all members of $P(X)$, it fixes $\beta$ and the result follows: $\Delta_{2}$ is then the trivial, and $\Delta$ is an inner automorphism of the group $\operatorname{Aut}(N)$.

ACKnowledgements. The author would like to thank Oleg Belegradek for helpful discussions and to thank the referee for the constructive comments on the manuscript.

\section{REFERENCES}

[1] S. Andreadakis. On the automorphisms of free groups and free nilpotent groups. Proc. London Math. Soc 15 (1965), 239-268.

[2] Yu. Bahturin. Basic structures of modern algebra. Mathematics and its Applications, 265. (Kluwer Academic Publishers Group, Dordrecht, 1993).

[3] M. Bridson and K. Vogtmann. Automorphisms of automorphism groups of free groups. J. Algebra. 229 (2000), 785-792.

[4] R. M. Bryant and C. K. Gupta. Automorphism groups of free nilpotent groups. Arch. Math. (Basel) 52 (1989), 313-320.

[5] R. M. Bryant and V. A. Roman'kov. The automorphism groups of relatively free algebras. J. Algebra 209 (1998), 713-723.

[6] R. G. Burns and L. Pi. Generators for the bounded automorphisms of infinite-rank free nilpotent groups. Bull. Austral. Math. Soc. 40 (1989), 175-187.

[7] J. Dixon and B. Mortimer. Permutation groups. Graduate Texts in Mathematics, 163. (Springer-Verlag, New York, 1996).

[8] J. Dyer and E. Formanek. The automorphism group of a free group is complete. J. London Math. Soc. 11 (1975), 181-190.

[9] J. Dyer and E. Formanek. Automorphism sequences of free nilpotent group of class two. Math. Proc. Camb. Phil. Soc. 79 (1976), 271-279.

[10] J. Dyer and E. Formanek. Characteristic subgroups and complete automorphism groups. Amer. J. Math. 99 (1977), 713-753.

[11] E. Formanek. Characterizing a free group in its automorphism group. J. Algebra. 133 (1990), 424-432.

[12] W. Hodges. Model Theory. (University Press, Cambridge, 1993).

[13] M. Kassabov. On the automorphism tower of free nilpotent groups. PhD. thesis. Yale University (2003).

[14] D. G. Khramtsov. Completeness of groups of outer automorphisms of free groups. Grouptheoretic investigations (Akad. Nauk SSSR Ural. Otdel., Sverdlovsk, 1990), pp. 128-143.

[15] W. Magnus, A. Karrass and D. Solitar. Combinatorial Group Theory. (Wiley, 1966). 
[16] H. Neumann. Varieties of Groups. (Springer-Verlag, 1967).

[17] V. Tolstykh. The automorphism tower of a free group. J. London Math. Soc. (2) 61 (2000), 423-440.

[18] V. Tolstykh. Free two-step nilpotent groups whose automorphism group is complete. Math. Proc. Cambridge Philos. Soc. 131 (2001), 73-90.

[19] V. Tolstykh. On the logical strength of the automorphism groups of free nilpotent groups. Contemp. Math. vol. 302 (AMS, Providence, 2002) pp. 113-120.

[20] V. Tolstykh. On the Bergman property for the automorphism groups of relatively free groups. J. London Math. Soc. (2) 73 (2006), 669-680.

Vladimir Tolstykh, Department of Mathematics, Yeditepe University, 34755 Kayişda Ği, Istanbul, Turkey

E-mail address: vtolstykh@yeditepe.edu.tr, tvlaa@rambler.ru 